ESAIM: COCV 27 (2021) 53

https://doi.org/10.1051/cocv/2021051
ESAIM: Control, Optimisation and Calculus of Variations

www.esaim-cocv.org

\title{
STABILIZATION OF PORT-HAMILTONIAN SYSTEMS BY NONLINEAR BOUNDARY CONTROL IN THE PRESENCE OF DISTURBANCES
}

\author{
JoChen SCHMID ${ }^{1,2, *}$ AND Hans ZwarT ${ }^{3,4}$
}

\begin{abstract}
In this paper, we are concerned with the stabilization of linear port-Hamiltonian systems of arbitrary order $N \in \mathbb{N}$ on a bounded 1-dimensional spatial domain $(a, b)$. In order to achieve stabilization, we couple the system to a dynamic boundary controller, that is, a controller that acts on the system only via the boundary points $a, b$ of the spatial domain. We use a nonlinear controller in order to capture the nonlinear behavior that realistic actuators often exhibit and, moreover, we allow the output of the controller to be corrupted by actuator disturbances before it is fed back into the system. What we show here is that the resulting nonlinear closed-loop system is input-to-state stable w.r.t. square-integrable disturbance inputs. In particular, we obtain uniform input-to-state stability for systems of order $N=1$ and a special class of nonlinear controllers, and weak input-to-state stability for systems of arbitrary order $N \in \mathbb{N}$ and a more general class of nonlinear controllers. Also, in both cases, we obtain convergence to 0 of all solutions as $t \rightarrow \infty$. Applications are given to vibrating strings and beams.
\end{abstract}

Mathematics Subject Classification. 93D15, 93D09, 93C20, 93D21, 35L65.

Received September 11, 2018. Accepted May 10, 2021.

\section{INTRODUCTION}

In this paper, we consider linear port-Hamiltonian systems of arbitrary order $N \in \mathbb{N}$ on a bounded 1dimensional spatial domain $(a, b)$. Such systems are described by a linear partial differential equation of the form

$$
\partial_{t} x(t, \zeta)=P_{N} \partial_{\zeta}^{N}(\mathcal{H}(\zeta) x(t, \zeta))+\cdots+P_{1} \partial_{\zeta}(\mathcal{H}(\zeta) x(t, \zeta))+P_{0} \mathcal{H}(\zeta) x(t, \zeta)
$$

Keywords and phrases: Input-to-state stability, infinite-dimensional systems, port-Hamiltonian systems, nonlinear boundary control, actuator disturbances.

${ }^{1}$ Institut für Mathematik, Universität Würzburg, 97074 Würzburg, Germany.

2 Fraunhofer Institute for Industrial Mathematics (ITWM), 67663 Kaiserslautern, Germany.

3 Department of Applied Mathematics, University of Twente, 7500 AE Enschede, The Netherlands.

${ }^{4}$ Department of Mechanical Engineering, Technische Universiteit Eindhoven, 5600 MB Eindhoven, The Netherlands.

* Corresponding author: jochen.schmid@itwm.fraunhofer.de 
for $t \in[0, \infty), \zeta \in(a, b)$, and the energy of such a system in the state $x(t, \cdot)$ is given by

$$
E(x(t, \cdot))=\frac{1}{2} \int_{a}^{b} x(t, \zeta)^{\top} \mathcal{H}(\zeta) x(t, \zeta) \mathrm{d} \zeta
$$

where $x(t, \zeta) \in \mathbb{R}^{m}, \mathcal{H}(\zeta) \in \mathbb{R}^{m \times m}$ is the energy density at $\zeta$, and $P_{0}, \ldots, P_{N} \in \mathbb{R}^{m \times m}$ are alternately skewsymmetric and symmetric matrices and $P_{N}$ is invertible. Simple examples of such systems are given by vibrating strings or beams. Also, many of the systems of linear conservation laws considered in [3], namely those of the special form

$$
\partial_{t} x(t, \zeta)=P \partial_{\zeta} x(t, \zeta) \quad(t \in[0, \infty), \zeta \in(a, b))
$$

with a $\zeta$-independent invertible matrix $P$, fall in the above class of linear port-Hamiltonian systems. What we are interested in here is the stabilization of such a system $\mathfrak{S}$ by means of dynamic boundary control, that is, by coupling the system to a dynamic controller $\mathfrak{S}_{c}$ that acts on the system only via the boundary points $a, b$ of the spatial domain $(a, b)$. Since realistic controllers often exhibit nonlinear behavior (due to nonquadratic potential energy or nonlinear damping terms, for instance), we want to work with nonlinear controllers - just like $[1,28,42,59]$. Since, moreover, realistic controllers are typically affected by external disturbances, we unlike $[1,28,42,59]$ - also want to incorporate such actuator disturbances which corrupt the output of the controller before it is fed back into the system. Coupling such a controller to the system $\mathfrak{S}$ by standard feedback interconnection

$$
y(t)=u_{c}(t) \quad \text { and } \quad-y_{c}(t)+d(t)=u(t)
$$

(with $u, y$ and $u_{c}, y_{c}$ being the in- and outputs of $\mathfrak{S}$ and $\mathfrak{S}_{c}$ respectively and with $d$ being the disturbance), we obtain a nonlinear closed-loop system $\tilde{\mathfrak{S}}$ with input $d$ and output $y$. A block diagram of this closed-loop system is shown in the following figure.

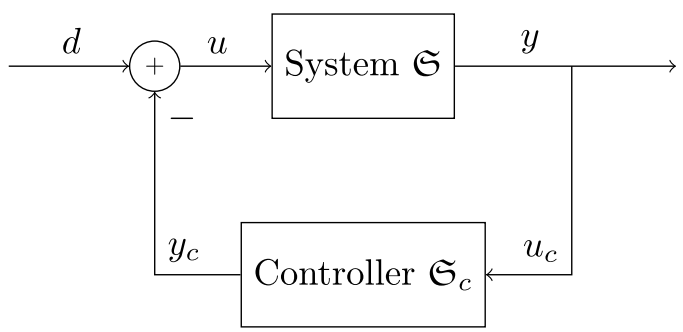

In this paper, we establish the input-to-state stability for the closed-loop system $\tilde{\mathfrak{S}}$ w.r.t. square-integrable disturbance inputs $d$. In rough terms, this means that $\tilde{x}_{*}:=0$ is an asymptotically stable equilibrium point of the undisturbed closed-loop system with $d=0$ and that this stability property is robust w.r.t. disturbances $d \neq 0$ in the sense that small disturbances affect the asymptotic stability only slightly (see (1.5) and (1.6) below). In more precise terms, the input-to-state stability of $\widetilde{\mathfrak{S}}$ w.r.t. square-integrable disturbance inputs $d$ means (i) that $\tilde{\mathfrak{S}}$, for every initial state $\tilde{x}_{0}$ and every square-integrable disturbance $d$, has a unique global (generalized) solution $\tilde{x}\left(\cdot, \tilde{x}_{0}, d\right)$ and (ii) that for all these (generalized) solutions, the following perturbed stability and attractivity estimates hold true:

$$
\begin{gathered}
\left\|\tilde{x}\left(t, \tilde{x}_{0}, d\right)\right\| \leq \underline{\sigma}\left(\left\|\tilde{x}_{0}\right\|\right)+\underline{\gamma}\left(\|d\|_{2}\right) \quad(t \in[0, \infty)) \\
\text { and } \\
\limsup _{t \rightarrow \infty}\left\|\tilde{x}\left(t, \tilde{x}_{0}, d\right)\right\| \leq \bar{\gamma}\left(\|d\|_{2}\right),
\end{gathered}
$$


where $\underline{\sigma}, \gamma, \bar{\gamma}$ are monotonically increasing comparison functions that are zero at 0 . According to whether the limit relation (1.6) holds (locally) uniformly or just pointwise w.r.t. $\tilde{x}_{0}$ and $d$, one speaks of uniform or weak input-to-state stability, respectively. Also, instead of uniform input-to-state stability one usually just speaks of input-to-state stability. In this paper, we show (i) that for a system $\mathfrak{S}$ of order $N=1$ and a special class of nonlinear controllers $\mathfrak{S}_{c}$, the resulting closed-loop system $\tilde{\mathfrak{S}}$ is uniformly input-to-state stable w.r.t. square-integrable disturbances $d$, and (ii) that for a system $\mathfrak{S}$ of arbitrary order $N \in \mathbb{N}$ and a more general class of nonlinear controllers $\mathfrak{S}_{c}$, the resulting closed-loop system $\tilde{\mathfrak{S}}$ is weakly input-to-state stable w.r.t. square-integrable disturbances $d$. In particular, we show in both cases that unique global (generalized) solutions $\tilde{x}\left(\cdot, \tilde{x}_{0}, d\right)$ exist for $\tilde{\mathfrak{S}}$. Additionally, we will see that in both cases every such solution converges to zero:

$$
\tilde{x}\left(t, \tilde{x}_{0}, d\right) \longrightarrow 0 \quad(t \rightarrow \infty)
$$

for every initial state $\tilde{x}_{0}$ and every square-integrable disturbance $d$. In all these results, we have to impose only mild assumptions on the system $\mathfrak{S}$ to be stabilized, namely an impedance-passivity condition and an approximate observability condition. We finally apply both our uniform and our weak input-to-state stability result to vibrating strings and beams (modeled according to Timoshenko).

In the literature, the stabilization of port-Hamiltonian systems has been considered so far, to the best of our knowledge, only in the case without actuator disturbances. In [1, 28, 42, 54, 59], no disturbances are considered at all, that is, the situation depicted above is considered in the special case where $d=0$. Stabilization of port-Hamiltonian systems of various degrees of generality is achieved by means of linear dynamic boundary controllers in [54] and by means of nonlinear dynamic boundary controllers in [1, 28, 42, 59]. In [51], sensor instead of actuator - disturbances are considered, that is, disturbances do occur in [51] but they corrupt the input of the controller instead of its output. It is shown in [51] that for a port-Hamiltonian system of the special form (1.3) with negative definite $P$ and a linear dynamic boundary controller, the resulting closed-loop system is uniformly input-to-state stable w.r.t. essentially bounded disturbance inputs $d$ (meaning that the perturbed stability and attractivity estimates (1.5) and (1.6) are satisfied with the 2-norm $\|d\|_{2}$ replaced by the $\infty$-norm $\|d\|_{\infty}$ of the disturbance).

In recent years, input-to-state stability of linear and non-linear partial differential equation systems parabolic and hyperbolic - has attracted a great deal of research, both in the case of distributed inputs (entering in the domain) and in the typically more challenging case of boundary inputs (entering at the boundary of the domain of the considered pde). See $[6,10,16,22-24,27,32,50,51,56-58]$, for instance. So far, the works establishing input-to-state stability of parabolic pde systems by far outnumber those on hyperbolic pde systems (like the port-Hamiltonian systems considered here). While the aforementioned papers deal with input-to-state stability w.r.t. a single equilibrium point of pde systems, a few papers $[8,49]$ also establish input-to-state stability w.r.t. attractors of such systems. Apart from the mentioned works on pde systems, also a lot of papers on the input-to-state stability of finite or infinite networks of ordinary or partial differential equation subsystems and on the input-to-state stability of time-delay systems have appeared in recent years. See [7, 25, 34, 35] and, respectively, [21, 36, 40] among many others. And finally, a multitude of papers have recently been published on fundamental characterizations and criteria for input-to-state stability [15, 17-19, 30, 31] and for versions of input-to-state stability like local [29], integral [15], strong [37], weak [45] and input-to-state practical stability [33], for instance. A nice survey of the state of the art of input-to-state stability for infinite-dimensional systems can be found in [36] - along with an extensive list of references on the topic.

We conclude the introduction with some remarks on the organization of the paper and on notational conventions used throughout the paper. Section 2 provides a detailed description of the setting with the precise assumptions on the system $\mathfrak{S}$ to be stabilized and the controller $\mathfrak{S}_{c}$ used for that purpose. In Section 3 we prove the solvability of the closed-loop system - first, in the classical sense for classical data $\left(\tilde{x}_{0}, d\right)$, and then in the generalized sense for general data $\left(\tilde{x}_{0}, d\right)$. In Section 4 we establish the main results of this paper, namely the uniform (Sect. 4.1) and the weak (Sect. 4.2) input-to-state stability of the closed-loop system. And finally, in Section 5 we present some applications of our general results. In order to get a quick overview of the core results in simplified form, the reader can consult the conference paper [43]. 
In the entire paper, $|\cdot|$ denotes the standard norm on $\mathbb{R}^{k}$ for every $k \in \mathbb{N}$. As usual, $\mathcal{K}, \mathcal{K}{ }_{\infty}, \mathcal{L}$ denote the following classes of comparison functions:

$$
\begin{gathered}
\mathcal{K}:=\{\gamma \in C([0, \infty),[0, \infty)): \gamma \text { strictly increasing with } \gamma(0)=0\} \\
\mathcal{K}_{\infty}:=\{\gamma \in \mathcal{K}: \gamma \text { unbounded }\} \\
\mathcal{L}:=\left\{\gamma \in C([0, \infty),[0, \infty)): \gamma \text { strictly decreasing with } \lim _{r \rightarrow \infty} \gamma(r)=0\right\}
\end{gathered}
$$

Also, $C_{c}^{2}\left([0, \infty), \mathbb{R}^{k}\right)$ denotes the subspace of $C^{2}\left([0, \infty), \mathbb{R}^{k}\right)$-functions $d$ with compact support in $[0, \infty)$, and for $d \in L^{2}\left([0, \infty), \mathbb{R}^{k}\right)$ we will use the following short-hand notations:

$$
\|d\|_{2}:=\|d\|_{L^{2}\left([0, \infty), \mathbb{R}^{k}\right)}, \quad\|d\|_{[0, t], 2}:=\left\|\left.d\right|_{[0, t]}\right\|_{L^{2}\left([0, t], \mathbb{R}^{k}\right)} .
$$

And finally, for a semigroup generator $A$ and bounded operators $B, C$ between appropriate spaces, the symbol $\mathfrak{S}(A, B, C)$ will stand for the state-linear system [5]

$$
x^{\prime}=A x+B u \quad \text { with } \quad y=C x,
$$

where the prime stands for the derivative w.r.t. time.

\section{SETTING}

\subsection{Setting: the system to be stabilized}

We consider a linear port-Hamiltonian system $\mathfrak{S}$ of order $N \in \mathbb{N}$ on a bounded interval $(a, b)$ with control and observation at the boundary $[2,9,12]$. Such a system evolves according to the following differential equation with boundary control and boundary observation conditions:

$$
\begin{array}{r}
x^{\prime}=\mathcal{A} x=P_{N} \partial_{\zeta}^{N}(\mathcal{H} x)+P_{N-1} \partial_{\zeta}^{N-1}(\mathcal{H} x)+\cdots+P_{1} \partial_{\zeta}(\mathcal{H} x)+P_{0} \mathcal{H} x \\
u(t)=\mathcal{B} x(t) \quad \text { and } \quad y(t)=\mathcal{C} x(t)
\end{array}
$$

and the energy of such a system in the state $x$ is given by

$$
E(x)=\frac{1}{2} \int_{a}^{b} x(\zeta)^{\top}(\mathcal{H} x)(\zeta) \mathrm{d} \zeta
$$

In these equations, $\zeta \mapsto \mathcal{H}(\zeta) \in \mathbb{R}^{m \times m}$ is a measurable matrix-valued function (the energy density) such that for some positive constants $\underline{m}, \bar{m}$ and almost all $\zeta \in(a, b)$

$$
0<\underline{m} \leq \mathcal{H}(\zeta) \leq \bar{m}<\infty
$$

and $P_{0}, P_{1}, \ldots, P_{N} \in \mathbb{R}^{m \times m}$ are matrices such that $P_{N}$ is invertible and $P_{1}, \ldots, P_{N}$ are alternately symmetric and skew-symmetric while $P_{0}$ is dissipative:

$$
P_{l}^{\top}=(-1)^{l+1} P_{l} \quad(l \in\{1, \ldots, N\}) \quad \text { and } \quad P_{0}^{\top}+P_{0} \leq 0 .
$$

(Strictly speaking, such systems should be called port-Hamilitonian only if $P_{0}$ is skew-symmetric. If $P_{0}$ is only dissipative, they could more precisely be called port-dissipative systems.) As the state space of $\mathfrak{S}$ one chooses 
$X:=L^{2}\left((a, b), \mathbb{R}^{m}\right)$ with norm $\|\cdot\|_{X}$ given by the system energy

$$
\frac{1}{2}\|x\|_{X}^{2}:=E(x)=\frac{1}{2} \int_{a}^{b} x(\zeta)^{\top}(\mathcal{H} x)(\zeta) \mathrm{d} \zeta .
$$

It is clear by (2.4) that the norm $\|\cdot\|_{X}$ is equivalent to the standard norm of $L^{2}\left((a, b), \mathbb{R}^{m}\right)$ and that it is induced by a scalar product which we denote by $\langle\cdot, \cdot\rangle_{X}$. As the domain of the linear differential operator $\mathcal{A}: D(\mathcal{A}) \subset X \rightarrow X$ from $(2.1)$ one chooses

$$
D(\mathcal{A}):=\left\{x \in X: \mathcal{H} x \in W^{N, 2}\left((a, b), \mathbb{R}^{m}\right) \text { and }\left.W_{B, 1}(\mathcal{H} x)\right|_{\partial}=0\right\}
$$

where $W_{B, 1} \in \mathbb{R}^{(m N-k) \times 2 m N}$ with $k \in\{1, \ldots, m N\}$ and where

$$
\left.(\mathcal{H} x)\right|_{\partial}:=\left(\begin{array}{c}
\left.(\mathcal{H} x)\right|_{\partial, b} \\
\left.(\mathcal{H} x)\right|_{\partial, a}
\end{array}\right),\left.\quad(\mathcal{H} x)\right|_{\partial, \zeta}:=\left((\mathcal{H} x)(\zeta)^{\top}, \partial_{\zeta}(\mathcal{H} x)(\zeta)^{\top}, \ldots, \partial_{\zeta}^{N-1}(\mathcal{H} x)(\zeta)^{\top}\right)^{\top} .
$$

In other words, the domain of $\mathcal{A}$ incorporates the zero boundary condition

$$
\left.W_{B, 1}(\mathcal{H} x)\right|_{\partial}=0
$$

which consists of $N m-k$ (scalar) equations and is linear in the boundary values of $(\mathcal{H} x), \partial_{\zeta}(\mathcal{H} x), \ldots, \partial_{\zeta}^{N-1}(\mathcal{H} x)$. Similarly, the boundary control and boundary observation conditions (2.2) consist of $k$ (scalar) equations each and are again linear in $\left.(\mathcal{H} x)\right|_{\partial}$, that is, the boundary control and boundary observation operators $\mathcal{B}, \mathcal{C}: D(\mathcal{A}) \rightarrow$ $\mathbb{R}^{k}$ from (2.2) are linear and of the form

$$
\mathcal{B} x:=\left.W_{B, 2}(\mathcal{H} x)\right|_{\partial} \quad \text { and } \quad \mathcal{C} x:=\left.W_{C}(\mathcal{H} x)\right|_{\partial}
$$

with matrices $W_{B, 2}, W_{C} \in \mathbb{R}^{k \times 2 m N}$. In all our results, we will impose the following additional impedancepassivity condition on $\mathfrak{S}$.

Condition 2.1. $\mathfrak{S}$ is impedance-passive, that is,

$$
\langle x, \mathcal{A} x\rangle_{X} \leq(\mathcal{B} x)^{\top} \mathcal{C} x \quad(x \in D(\mathcal{A})) .
$$

It follows from Condition 2.1 by virtue of [14] (Cor. 2.3) that

$$
A:=\left.\mathcal{A}\right|_{D(\mathcal{A}) \cap \operatorname{ker} \mathcal{B}}
$$

is a contraction semigroup generator on $X$ and that the matrix $W_{B}:=\left(W_{B, 1}^{\top}, W_{B, 2}^{\top}\right)^{\top} \in \mathbb{R}^{m N \times 2 m N}$ has full row rank. In particular, $\mathcal{A}$ and $\mathcal{B}$ define a boundary control system [12] (by the same arguments as for Thm. 11.3.2 of [12] and by Lem. A.3 of [26]), so that for every initial state $x_{0} \in D(\mathcal{A})$ and every control input $u \in C^{2}\left([0, \infty), \mathbb{R}^{k}\right)$ with $u(0)=\mathcal{B} x_{0}$, the system (2.1) and (2.2) has a unique global classical solution

$$
x\left(\cdot, x_{0}, u\right) \in C^{1}([0, \infty), X) .
$$

Also, along every such classical solution, the following energy dissipation inequality is satisfied by virtue of (2.8):

$$
E_{x}^{\prime}(t)=\langle x(t), \mathcal{A} x(t)\rangle_{X} \leq(\mathcal{B} x(t))^{\top} \mathcal{C} x(t)=u(t)^{\top} y(t) \quad(t \in[0, \infty)),
$$

where $E_{x}(t):=E(x(t))$ and $x(t):=x\left(t, x_{0}, u\right)$ and $y(t):=y\left(t, x_{0}, u\right):=\mathcal{C} x\left(t, x_{0}, u\right)$. 


\subsection{Setting: the controller}

As the controller $\mathfrak{S}_{c}$ to stabilize $\mathfrak{S}$ we choose a finite-dimensional nonlinear system which evolves according to the ordinary differential equation (with input $u_{c}$ and output $y_{c}$ )

$$
\begin{gathered}
v^{\prime}=\left(\begin{array}{c}
v_{1}^{\prime} \\
v_{2}^{\prime}
\end{array}\right)=\left(\begin{array}{c}
K v_{2} \\
-\nabla \mathcal{P}\left(v_{1}\right)-\mathcal{R}\left(K v_{2}\right)+B_{c} u_{c}
\end{array}\right) \\
y_{c}=B_{c}^{\top} K v_{2}+S_{c} u_{c}
\end{gathered}
$$

and whose energy in the state $v=\left(v_{1}, v_{2}\right) \in \mathbb{R}^{2 m_{c}}$ is given by

$$
E_{c}(v):=\mathcal{P}\left(v_{1}\right)+\frac{1}{2} v_{2}^{\top} K v_{2}
$$

(potential energy plus kinetic energy). In these equations, $K \in \mathbb{R}^{m_{c} \times m_{c}}, B_{c} \in \mathbb{R}^{m_{c} \times k}, S_{c} \in \mathbb{R}^{k \times k}$ represent a generalized mass matrix, an input matrix, and a direct feedthrough matrix respectively and they are such that

$$
K>0 \quad \text { and } \quad S_{c} \geq \varsigma>0 \quad\left(\varsigma:=\min \sigma\left(S_{c}\right)\right)
$$

Additionally, the potential energy $\mathcal{P}: \mathbb{R}^{m_{c}} \rightarrow[0, \infty)$ is differentiable such that $\nabla \mathcal{P}$ is locally Lipschitz continuous and $\mathcal{P}(0)=0$ and the damping function $\mathcal{R}: \mathbb{R}^{m_{c}} \rightarrow \mathbb{R}^{m_{c}}$ is locally Lipschitz continuous such that $\mathcal{R}(0)=0$. As the norm on the controller state space $V:=\mathbb{R}^{2 m_{c}}$ we choose $|\cdot|_{V}$ defined by

$$
|v|_{V}^{2}=\left|\left(v_{1}, v_{2}\right)\right|_{V}^{2}:=\left|v_{1}\right|^{2}+v_{2}^{\top} K v_{2}
$$

which is obviously equivalent to the standard norm on $\mathbb{R}^{2 m_{c}}$ and is induced by a scalar product $\langle\cdot, \cdot\rangle_{V} \cdot$ In all our results, we will impose the following additional conditions on the controller $\mathfrak{S}_{c}$.

Condition 2.2. $\quad$ (i) $\mathcal{P}$ is positive definite and radially unbounded, that is, $\mathcal{P}\left(v_{1}\right)>0$ for all $v_{1} \in \mathbb{R}^{m_{c}} \backslash\{0\}$ and $\mathcal{P}\left(v_{1}\right) \longrightarrow \infty$ as $\left|v_{1}\right| \rightarrow \infty$

(ii) $\mathcal{R}$ is damping, that is, $v_{2}^{\top} \mathcal{R}\left(v_{2}\right) \geq 0$ for all $v_{2} \in \mathbb{R}^{m_{c}}$.

It follows from Condition 2.2 (ii) and from (2.15) that the controller system $\mathfrak{S}_{c}$ is passive (in fact, strictly input-passive) w.r.t. the storage function $E_{c}$.

\subsection{Setting: the closed-loop system}

Coupling $\mathfrak{S}$ and $\mathfrak{S}_{c}$ by standard feedback interconnection

$$
y(t)=u_{c}(t) \quad \text { and } \quad-y_{c}(t)+d(t)=u(t)
$$

we obtain the closed-loop system $\tilde{\mathfrak{S}}$ described by the following evolution equation and boundary input and output conditions:

$$
\begin{aligned}
\tilde{x}^{\prime}=\tilde{\mathcal{A}} \tilde{x}+\tilde{f}(\tilde{x}) \\
d(t)=\tilde{\mathcal{B}} \tilde{x}(t) \quad \text { and } \quad y(t)=\tilde{\mathcal{C}} \tilde{x}(t) .
\end{aligned}
$$

Its state space is the Hilbert space $\tilde{X}:=X \times V=X \times \mathbb{R}^{2 m_{c}}$ with norm $\|\cdot\|=\|\cdot\|_{\tilde{X}}$ defined by

$$
\|\tilde{x}\|^{2}=\|(x, v)\|^{2}:=\|x\|_{X}^{2}+|v|_{V}^{2}
$$


and the energy of the closed-loop system in the state $\tilde{x}=(x, v) \in \tilde{X}$ is

$$
\tilde{E}(\tilde{x}):=E(x)+E_{c}(v)=\frac{1}{2}\|x\|_{X}^{2}+\mathcal{P}\left(v_{1}\right)+\frac{1}{2} v_{2}^{\top} K v_{2}
$$

It follows from Condition 2.2 (i) (taking into account Lemma 2.5 of [4]) that the energy $\tilde{E}$ is equivalent to the norm $\|\cdot\|$ of $\tilde{X}$ in the following sense: there exist $\underline{\psi}, \bar{\psi} \in \mathcal{K}_{\infty}$ such that for all $\tilde{x} \in \tilde{X}$

$$
\underline{\psi}(\|\tilde{x}\|) \leq \tilde{E}(\tilde{x}) \leq \bar{\psi}(\|\tilde{x}\|) .
$$

In the equations above, the linear and nonlinear operators $\tilde{\mathcal{A}}: D(\tilde{\mathcal{A}}) \rightarrow \tilde{X}$ and $\tilde{f}: \tilde{X} \rightarrow \tilde{X}$ with $D(\tilde{\mathcal{A}}):=$ $D(\mathcal{A}) \times \mathbb{R}^{2 m_{c}}$ are given respectively by

$$
\tilde{\mathcal{A}} \tilde{x}:=\left(\begin{array}{c}
\mathcal{A} x \\
K v_{2} \\
-v_{1}+B_{c} \mathcal{C} x
\end{array}\right) \quad \text { and } \quad \tilde{f}(\tilde{x}):=\left(\begin{array}{c}
0 \\
0 \\
v_{1}-\nabla \mathcal{P}\left(v_{1}\right)-\mathcal{R}\left(K v_{2}\right)
\end{array}\right)
$$

and the linear boundary input and output operators $\tilde{\mathcal{B}}, \tilde{\mathcal{C}}: D(\tilde{\mathcal{A}}) \rightarrow \mathbb{R}^{k}$ are given by

$$
\tilde{\mathcal{B}} \tilde{x}:=\mathcal{B} x+B_{c}^{\top} K v_{2}+S_{c} \mathcal{C} x, \quad \tilde{\mathcal{C}} \tilde{x}:=\mathcal{C} x
$$

for $\tilde{x}=\left(x, v_{1}, v_{2}\right) \in D(\tilde{\mathcal{A}})$. In our solvability and stability results, we will need the following semigroup generation and compact resolvent result. A version of this lemma is stated in [42] (Lem. 3), namely for the special case $N=1$, but without proof and with an unnecessary full-rank condition on $W_{B}:=\left(W_{B, 1}^{\top}, W_{B, 2}^{\top}\right)^{\top}$. Another version of the lemma can be found in [53] (Thm. 5.8), namely for the special case of impedance-energy-preserving openloop systems and strictly positive real linear controllers (and, again, with an unnecessary full-rank condition on $\left.W:=\left(W_{B}^{\top}, W_{C}^{\top}\right)^{\top}\right)$.

Lemma 2.3. If Condition 2.1 and 2.2 are satisfied, then

(i) the operator $\tilde{A}:=\left.\tilde{\mathcal{A}}\right|_{D(\tilde{\mathcal{A}}) \cap \operatorname{ker} \tilde{\mathcal{B}}}$ is a contraction semigroup generator on $\tilde{X}$ with compact resolvent

(ii) $\tilde{f}$ is Lipschitz continuous on bounded subsets of $\tilde{X}$.

Proof. Since assertion (ii) is clear by our assumptions on $\mathcal{P}$ and $\mathcal{R}$, we have only to prove assertion (i). We do so in three steps. As a first step, we observe that $\tilde{A}$ is dissipative in $\tilde{X}$. Indeed, for every $\tilde{x}=(x, v) \in D(\tilde{A})$ we have $x \in D(\mathcal{A})$ and $B_{c}^{\top} K v_{2}=-\mathcal{B} x-S_{c} \mathcal{C} x$ and hence

$$
\langle\tilde{x}, \tilde{A} \tilde{x}\rangle_{\tilde{X}}=\langle x, \mathcal{A} x\rangle_{X}+v_{1}^{\top} K v_{2}+v_{2}^{\top} K\left(-v_{1}+B_{c} \mathcal{C} x\right) \leq(\mathcal{B} x)^{\top} \mathcal{C} x+\left(B_{c}^{\top} K v_{2}\right)^{\top} \mathcal{C} x \leq 0
$$

where the last two inequalities follow from (2.8) and (2.15). Consequently, $\tilde{A}$ is dissipative in $\tilde{X}$, as desired.

As a second step, we show that $\tilde{A}-\lambda$ is surjective onto $\tilde{X}$ for every $\lambda \in(0, \infty)$ with

$$
2 \lambda \geq A_{c}+A_{c}^{\top} \quad\left(A_{c}:=\left(\begin{array}{cc}
0 & K \\
-I & 0
\end{array}\right) \in \mathbb{R}^{2 m_{c} \times 2 m_{c}}\right) .
$$

So let $\lambda \in(0, \infty)$ as in $(2.20)$ and let $\tilde{y}=(y, w) \in \tilde{X}$. We then have to find an $\tilde{x}=(x, v) \in D(\tilde{A})$, that is, an $\tilde{x} \in \tilde{X}$ with

$$
x \in D(\mathcal{A}) \quad \text { and }\left.\quad\left(W_{B, 2}+S_{c} W_{C}\right)(\mathcal{H} x)\right|_{\partial}+B_{c}^{\top} K v_{2}=0,
$$


such that $(\tilde{A}-\lambda) \tilde{x}=\tilde{y}$, that is,

$$
(\mathcal{A}-\lambda) x=y \quad \text { and } \quad\left(A_{c}-\lambda\right) v+\left(\begin{array}{c}
0 \\
B_{c} \mathcal{C} x
\end{array}\right)=w
$$

Since $A_{c}-\lambda$ is invertible for $\lambda \in(0, \infty)$, finding an $\tilde{x} \in \tilde{X}$ with (2.21) and (2.22) is equivalent to finding $x \in X$ such that

$$
\begin{gathered}
x \in D(\mathcal{A}) \quad \text { and } \quad \mathcal{B}^{\prime} x=B_{c}^{\top} K\left(\begin{array}{ll}
0 & I
\end{array}\right)\left(\lambda-A_{c}\right)^{-1} w \\
(\mathcal{A}-\lambda) x=y,
\end{gathered}
$$

where $\mathcal{B}^{\prime} x:=\left.\left(W_{B, 2}+G_{\lambda} W_{C}\right)(\mathcal{H} x)\right|_{\partial}$ and

$$
\begin{gathered}
G_{\lambda}:=S_{c}+B_{c}^{\top} K\left(\begin{array}{ll}
0 & I
\end{array}\right)\left(\lambda-A_{c}\right)^{-1}\left(\begin{array}{c}
0 \\
B_{c}
\end{array}\right)=S_{c}+\left(\begin{array}{c}
0 \\
B_{c}
\end{array}\right)^{\top} L^{\top}\left(\lambda-A_{c}\right)^{-1} L\left(\begin{array}{c}
0 \\
B_{c}
\end{array}\right) \\
\left(L:=\left(\begin{array}{cc}
K^{1 / 2} & 0 \\
0 & K^{1 / 2}
\end{array}\right)\right) .
\end{gathered}
$$

(In the last equation we used that $L$ commutes with $A_{c}$.) In order to find an $\tilde{x}$ with (2.23) and (2.24), we will show that the port-Hamiltonian operator $A^{\prime}:=\left.\mathcal{A}\right|_{D\left(A^{\prime}\right)}$ with domain

$$
\begin{gathered}
D\left(A^{\prime}\right):=D(\mathcal{A}) \cap \operatorname{ker} \mathcal{B}^{\prime} \\
=\left\{x \in X: \mathcal{H} x \in W^{N, 2}\left((a, b), \mathbb{R}^{m}\right),\left.W_{B, 1}(\mathcal{H} x)\right|_{\partial}=0 \text { and }\left.\left(W_{B, 2}+G_{\lambda} W_{C}\right)(\mathcal{H} x)\right|_{\partial}=0\right\}
\end{gathered}
$$

generates a contraction semigroup on $X$. So let $x \in D\left(A^{\prime}\right)$ and $u:=\left.W_{B, 2}(\mathcal{H} x)\right|_{\partial}$ and $y:=\left.W_{C}(\mathcal{H} x)\right|_{\partial}$, then $x \in D(\mathcal{A})$ and $u+G_{\lambda} y=0$ and hence

$$
\left\langle x, A^{\prime} x\right\rangle_{X}=\langle x, \mathcal{A} x\rangle_{X} \leq u^{\top} y=-\frac{1}{2} y^{\top}\left(G_{\lambda}+G_{\lambda}^{\top}\right) y
$$

by virtue of (2.8). It follows from (2.15) and (2.20) that

$$
G_{\lambda}+G_{\lambda}^{\top}=2 S_{c}+\left(\begin{array}{c}
0 \\
B_{c}
\end{array}\right)^{\top} L^{\top}\left(\lambda-A_{c}\right)^{-1}\left(2 \lambda-\left(A_{c}+A_{c}^{\top}\right)\right)\left(\lambda-A_{c}^{\top}\right)^{-1} L\left(\begin{array}{c}
0 \\
B_{c}
\end{array}\right) \geq 0
$$

and thus (2.26) yields the dissipativity of $A^{\prime}$ in $X$. So, by the characterization of the contraction semigroup generator property for port-Hamiltonian operators from [14] (Cor. 2.3), $A^{\prime}$ is a contraction semigroup generator on $X$ and, moreover, the boundary matrix

$$
W^{\prime}:=\left(\begin{array}{c}
W_{B, 1} \\
W_{B, 2}+G_{\lambda} W_{C}
\end{array}\right) \in \mathbb{R}^{m N \times 2 m N}
$$

associated with $A^{\prime}$ has full row rank. In particular, $\mathcal{A}$ and $\mathcal{B}^{\prime}$ define a boundary control system by the same arguments as for Theorem 11.3.2 from [12] and by Lemma A.3 from [26], and hence there exists an operator $B^{\prime} \in L(U, X)$ with $U:=\mathbb{R}^{k}$ such that $B^{\prime} U \subset D(\mathcal{A})=D\left(\mathcal{B}^{\prime}\right)$ and $\mathcal{A} B^{\prime} \in L(U, X)$ and

$$
\mathcal{B}^{\prime} B^{\prime} u=u \quad(u \in U) .
$$


With these preliminary considerations about $A^{\prime}$ we can now finally prove the existence of an $x \in X$ with (2.23) and (2.24) and hence the surjectivity of $\tilde{A}-\lambda$. Indeed, with the ansatz

$$
x=x^{\prime}+h \quad\left(x^{\prime} \in D\left(A^{\prime}\right) \text { and } h \in D(\mathcal{A})\right),
$$

the conditions (2.23) and (2.24) become equivalent to

$$
\begin{aligned}
\mathcal{B}^{\prime} h & =B_{c}^{\top} K\left(\begin{array}{ll}
0 & I
\end{array}\right)\left(\lambda-A_{c}\right)^{-1} w \\
\left(A^{\prime}-\lambda\right) x^{\prime} & =y-(\mathcal{A}-\lambda) h .
\end{aligned}
$$

And since by $(2.27) \mathcal{B}^{\prime}$ is surjective and $A^{\prime}$ is a contraction semigroup generator, we really can find $h \in D\left(\mathcal{B}^{\prime}\right)=$ $D(\mathcal{A})$ and $x^{\prime} \in D\left(A^{\prime}\right)$ such that $(2.29)$ and (2.30) are satisfied, as desired.

As a third step, we show that $\tilde{A}$ has compact resolvent. So let $\lambda \in(0, \infty)$ with $(2.20)$. Also, let $\left(\tilde{y}_{n}\right)=\left(y_{n}, w_{n}\right)$ be a bounded sequence in $\tilde{X}$ and write

$$
\left(\begin{array}{l}
x_{n} \\
v_{n}
\end{array}\right)=\tilde{x}_{n}:=(\tilde{A}-\lambda)^{-1} \tilde{y}_{n}=(\tilde{A}-\lambda)^{-1}\left(\begin{array}{c}
y_{n} \\
w_{n}
\end{array}\right) \quad(n \in \mathbb{N})
$$

It then follows by $(2.28),(2.29),(2.30),(2.27)$ that $x_{n}=x_{n}^{\prime}+h_{n}$ with

$$
\begin{aligned}
& h_{n}=B^{\prime}\left(B_{c}^{\top} K\left(\begin{array}{ll}
0 & I
\end{array}\right)\left(\lambda-A_{c}\right)^{-1} w_{n}\right) \\
& x_{n}^{\prime}=\left(A^{\prime}-\lambda\right)^{-1}\left(y_{n}-(\mathcal{A}-\lambda) B^{\prime}\left(B_{c}^{\top} K\left(\begin{array}{ll}
0 & I
\end{array}\right)\left(\lambda-A_{c}\right)^{-1} w_{n}\right)\right) .
\end{aligned}
$$

Since $\left(\tilde{y}_{n}\right)=\left(y_{n}, w_{n}\right)$ is bounded and $\mathcal{A} B^{\prime} \in L(U, X)$, the sequences

$$
\left(w_{n}\right), \quad\left(v_{n}\right), \quad\left(y_{n}-(\mathcal{A}-\lambda) B^{\prime}\left(B_{c}^{\top} K(0 \quad I)\left(\lambda-A_{c}\right)^{-1} w_{n}\right)\right)
$$

are bounded as well. It follows by the finite-dimensionality of $\mathbb{R}^{2 m_{c}}$ and the compactness of $\left(A^{\prime}-\lambda\right)^{-1}$ (Thm. 2.28 in [53]) that there exists a subsequence $\left(n_{k}\right)$ sucht that $\left(w_{n_{k}}\right)$ and $\left(v_{n_{k}}\right)$ converge in $\mathbb{R}^{2 m_{c}}$ and such that $\left(x_{n_{k}}^{\prime}\right)$ converges in $X$. Consequently, $\left(\tilde{x}_{n_{k}}\right)$ is convergent as well, and we are done.

\section{Solvability OF THE ClOSED-LOOP SYSTEM}

In this section, we show that under suitable conditions the initial value problem

$$
\begin{aligned}
\tilde{x}^{\prime}=\tilde{\mathcal{A}} \tilde{x}+\tilde{f}(\tilde{x}) \quad & \text { and } \quad \tilde{x}(0)=\tilde{x}_{0} \\
d(t)=\tilde{\mathcal{B}} \tilde{x}(t) &
\end{aligned}
$$

of the closed-loop system has a global solution for suitable initial values $\tilde{x}_{0}$ and disturbance inputs $d$. We will achieve this by applying the standard theory of semilinear evolution equations from [39]. As it stands, however, the closed-loop equation (3.1) is not a semilinear evolution equation in the sense of [39] because, for one thing, the linear part $\tilde{\mathcal{A}}$ of the differential equation is not a semigroup generator and because, for another thing, in addition to the differential equation the side condition

$$
d(t)=\tilde{\mathcal{B}} \tilde{x}(t)
$$


occurs. A way out of this difficulty is to impose the following extra condition, which is easily seen to be satisfied if, for instance,

$$
W:=\left(\begin{array}{l}
W_{B} \\
W_{C}
\end{array}\right) \in \mathbb{R}^{(m N+k) \times 2 m N} \quad \text { with } \quad W_{B}:=\left(\begin{array}{l}
W_{B, 1} \\
W_{B, 2}
\end{array}\right)
$$

is a matrix of full row rank $m N+k$.

Condition 3.1. $\tilde{\mathcal{B}}$ is a surjective linear map from $D(\tilde{\mathcal{A}})$ onto $\mathbb{R}^{k}$.

With the help of this extra condition, we can turn (3.1) into a truly semilinear evolution equation in the sense of [39]. In fact, Condition 3.1 implies the existence of a linear right-inverse $\tilde{R}: \mathbb{R}^{k} \rightarrow D(\tilde{\mathcal{A}})$ of $\tilde{\mathcal{B}}$, that is,

$$
\tilde{\mathcal{B}} \tilde{R} d=d
$$

for all $d \in \mathbb{R}^{k}$. We can thus perform the transformation

$$
\tilde{\xi}(t)=\tilde{x}(t)-\tilde{R} d(t)
$$

which is well-known from linear boundary control problems [5, 11, 12]. Via this transformation, the classical solutions $\tilde{x}$ of (3.1) are in one-to-one correspondence - for continuously differentiable disturbances $d$ - with the classical solutions $\tilde{\xi}$ of

$$
\tilde{\xi}^{\prime}=\tilde{A} \tilde{\xi}+\tilde{f}(\tilde{\xi}+\tilde{R} d(t))+\tilde{\mathcal{A}} \tilde{R} d(t)-\tilde{R} d^{\prime}(t) \quad \text { and } \quad \tilde{\xi}(0)=\tilde{x}_{0}-\tilde{R} d(0) .
$$

And this is now, in view of Lemma 2.3, a truly semilinear evolution equation (with an explicitly time-dependent nonlinearity). We can therefore apply standard semilinear theory to obtain classical solvability of (3.1) for sufficiently regular $\tilde{x}_{0}$ and $d$ (Sect. 3.1), and, by a suitable density and approximation argument, we then also obtain generalized solvability of (3.1) for sufficiently irregular $\tilde{x}_{0}$ and $d$ (Sect. 3.2).

\subsection{Solvability in the classical sense}

In this section, we show that for sufficiently regular initial states $\tilde{x}_{0}$ and disturbances $d$, namely for

$$
\left(\tilde{x}_{0}, d\right) \in \mathcal{D}:=\left\{\left(\tilde{x}_{0}, d\right) \in D(\tilde{\mathcal{A}}) \times C_{c}^{2}\left([0, \infty), \mathbb{R}^{k}\right): d(0)=\tilde{\mathcal{B}} \tilde{x}_{0}\right\}
$$

(set of classical data), the closed-loop equation (3.1) has a classical solution existing globally in time. A classical solution of (3.1) is a continuously differentiable function $\tilde{x}: J \rightarrow \tilde{X}$ on an interval $J \subset[0, \infty)$ containing 0 such that for every $t \in J$ one has (i) that $\tilde{x}(t) \in D(\tilde{\mathcal{A}})$ and (ii) that

$$
\begin{gathered}
\tilde{x}^{\prime}(t)=\tilde{\mathcal{A}} \tilde{x}(t)+\tilde{f}(\tilde{x}(t)) \quad \text { and } \quad \tilde{x}(0)=\tilde{x}_{0} \\
d(t)=\tilde{\mathcal{B}} \tilde{x}(t) .
\end{gathered}
$$

Theorem 3.2. Suppose that Conditions 2.1, 2.2, 3.1 are satisfied. Then

(i) for every $\left(\tilde{x}_{0}, d\right) \in \mathcal{D}$ the closed-loop equation (3.1) has a unique global classical solution

$$
\tilde{x}\left(\cdot, \tilde{x}_{0}, d\right) \in C^{1}([0, \infty), \tilde{X})
$$

and the output $y\left(\cdot, \tilde{x}_{0}, d\right):=\tilde{\mathcal{C}} \tilde{x}\left(\cdot, \tilde{x}_{0}, d\right)$ is a continuous function from $[0, \infty)$ to $\mathbb{R}^{k}$ 
(ii) there exist $\underline{\sigma}, \underline{\gamma} \in \mathcal{K}$ such that for every $\left(\tilde{x}_{0}, d\right) \in \mathcal{D}$

$$
\left\|\tilde{x}\left(t, \tilde{x}_{0}, d\right)\right\| \leq \underline{\sigma}\left(\left\|\tilde{x}_{0}\right\|\right)+\underline{\gamma}\left(\|d\|_{[0, t], 2}\right) \quad(t \in[0, \infty)) .
$$

Proof. As a first step, we show that for every $\left(\tilde{x}_{0}, d\right) \in \mathcal{D}$ the initial value problem (3.1) has a unique maximal classical solution $\tilde{x}\left(\cdot, \tilde{x}_{0}, d\right)$ on some half-open interval $\left[0, T_{\tilde{x}_{0}, d}\right)$. So let $\left(\tilde{x}_{0}, d\right) \in \mathcal{D}$. As is easily verified, a function $\tilde{x}$ is a maximal classical solution of (3.1) if and only if the function $\tilde{x}-\tilde{R} d$ is a maximal classical solution of (3.4). We therefore have only to show that (3.4) has a unique maximal classical solution existing on some half-open interval of the form $[0, T)$. And, in view of Lemma 2.3, this immediately follows by the standard solvability results for semilinear evolution equations in reflexive spaces (Thms. 6.1.4 and 6.1.6 in [39]). In particular, it follows by variation of constants that for every $\left(\tilde{x}_{0}, d\right) \in \mathcal{D}$ the corresponding maximal classical solution $\tilde{x}=\tilde{x}\left(\cdot, \tilde{x}_{0}, d\right)$ satisfies the following integral equation:

$$
\tilde{x}(t)=\mathrm{e}^{\tilde{A} t} \tilde{x}_{0}+\int_{0}^{t} \mathrm{e}^{\tilde{A}(t-s)} \tilde{f}(\tilde{x}(s)) \mathrm{d} s+\Phi_{t}(d)
$$

for every $t \in\left[0, T_{\tilde{x}_{0}, d}\right)$, where

$$
\Phi_{t}(d):=-\mathrm{e}^{\tilde{A} t} \tilde{R} d(0)+\tilde{R} d(t)+\int_{0}^{t} \mathrm{e}^{\tilde{A}(t-s)}\left(\tilde{\mathcal{A}} \tilde{R} d(s)-\tilde{R} d^{\prime}(s)\right) \mathrm{d} s .
$$

As a second step, we show that for every $\left(\tilde{x}_{0}, d\right) \in \mathcal{D}$ the output $y\left(\cdot, \tilde{x}_{0}, d\right):=\tilde{\mathcal{C}} \tilde{x}\left(\cdot, \tilde{x}_{0}, d\right)$ is a continuous function from $\left[0, T_{\tilde{x}_{0}, d}\right)$ to $\mathbb{R}^{k}$. So let $\left(\tilde{x}_{0}, d\right) \in \mathcal{D}$, write $(x, v):=\tilde{x}:=\tilde{x}\left(\cdot, \tilde{x}_{0}, d\right)$, and let

$$
\|\cdot\|_{\mathcal{A}}:=\left(\|\cdot\|_{X}^{2}+\|\mathcal{A} \cdot\|_{X}^{2}\right)^{1 / 2}
$$

denote the graph norm of $\mathcal{A}$. Since $\tilde{x}$ is a classical solution of (3.1), the functions $t \mapsto x(t)$ and $t \mapsto \mathcal{A} x(t)=x^{\prime}(t)$ are continuous from $\left[0, T_{\tilde{x}_{0}, d}\right)$ to $X$ and therefore

$$
\left[0, T_{\tilde{x}_{0}, d}\right) \ni t \mapsto x(t) \in\left(D(\mathcal{A}),\|\cdot\|_{\mathcal{A}}\right)
$$

is continuous. Since, moreover,

$$
\underline{c}\|\mathcal{H} \xi\|_{W^{N, 2}} \leq\|\xi\|_{\mathcal{A}} \leq \bar{c}\|\mathcal{H} \xi\|_{W^{N, 2}} \quad(\xi \in D(\mathcal{A}))
$$

for some positive constants $\underline{c}, \bar{c}$ (Lem. 3.2.3 in [2]), the map

$$
D(\mathcal{A}) \ni \xi \mapsto \mathcal{C} \xi=\left.W_{C}(\mathcal{H} \xi)\right|_{\partial} \in \mathbb{R}^{k}
$$

is continuous as well. Combining now (3.12) and (3.13), the second step follows.

As a third step, we show that there exist $\underline{\sigma}, \underline{\gamma} \in \mathcal{K}$ such that for every $\left(\tilde{x}_{0}, d\right) \in \mathcal{D}$

$$
\left\|\tilde{x}\left(t, \tilde{x}_{0}, d\right)\right\| \leq \underline{\sigma}\left(\left\|\tilde{x}_{0}\right\|\right)+\underline{\gamma}\left(\|d\|_{[0, t], 2}\right)
$$

for all $t \in\left[0, T_{\tilde{x}_{0}, d}\right)$. So let $\left(\tilde{x}_{0}, d\right) \in \mathcal{D}$ and write $(x, v):=\tilde{x}\left(\cdot, \tilde{x}_{0}, d\right)$ and $y:=y\left(\cdot, \tilde{x}_{0}, d\right)=\mathcal{C} x$. Since $\tilde{x}\left(\cdot, \tilde{x}_{0}, d\right)$ is a classical solution by the first step, the function

$$
s \mapsto \tilde{E}_{\tilde{x}}(s):=\tilde{E}\left(\tilde{x}\left(s, \tilde{x}_{0}, d\right)=\frac{1}{2}\|x(s)\|_{X}^{2}+\mathcal{P}\left(v_{1}(s)\right)+\frac{1}{2} v_{2}(s)^{\top} K v_{2}(s)\right.
$$


is continuously differentiable and its derivative satisfies

$$
\begin{aligned}
\tilde{E}_{\tilde{x}}^{\prime}(s) & =\langle x(s), \mathcal{A} x(s)\rangle_{X}-\left(K v_{2}(s)\right)^{\top} \mathcal{R}\left(K v_{2}(s)\right)+\left(B_{c}^{\top} K v_{2}(s)\right)^{\top} y(s) \\
& =\langle x(s), \mathcal{A} x(s)\rangle_{X}-\left(K v_{2}(s)\right)^{\top} \mathcal{R}\left(K v_{2}(s)\right)+d(s)^{\top} y(s)-(\mathcal{B} x(s))^{\top} y(s)-y(s)^{\top} S_{c} y(s)
\end{aligned}
$$

for all $s \in\left[0, T_{\tilde{x}_{0}, d}\right)$. With the help of Condition 2.1 and 2.2 we therefore get that

$$
\begin{aligned}
\tilde{E}_{\tilde{x}}^{\prime}(s) & \leq|d(s)||y(s)|-\left(K v_{2}(s)\right)^{\top} \mathcal{R}\left(K v_{2}(s)\right)-\varsigma|y(s)|^{2} \\
& \leq \frac{\alpha}{2}|d(s)|^{2}+\frac{1}{2 \alpha}|y(s)|^{2}-\varsigma|y(s)|^{2}-\left(K v_{2}(s)\right)^{\top} \mathcal{R}\left(K v_{2}(s)\right) \leq \frac{\alpha}{2}|d(s)|^{2}+\frac{1}{2 \alpha}|y(s)|^{2}-\varsigma|y(s)|^{2}
\end{aligned}
$$

for all $s \in\left[0, T_{\tilde{x}_{0}, d}\right)$ and arbitrary $\alpha \in(0, \infty)$, where $\varsigma$ as in $(2.15)$ is the smallest eigenvalue of $S_{c}$. Choosing now $\alpha:=1 /(2 \varsigma)$, we see from (3.16) by integration that

$$
\tilde{E}\left(\tilde{x}\left(t, \tilde{x}_{0}, d\right)\right) \leq \tilde{E}\left(\tilde{x}_{0}\right)+\frac{1}{4 \varsigma}\|d\|_{[0, t], 2}^{2}
$$

for every $t \in\left[0, T_{\tilde{x}_{0}, d}\right)$. Since $\tilde{E}$ is equivalent to the norm of $\tilde{X}$ by (2.18), we further conclude that

$$
\left\|\tilde{x}\left(t, \tilde{x}_{0}, d\right)\right\| \leq \underline{\psi}^{-1}\left(\bar{\psi}\left(\left\|\tilde{x}_{0}\right\|\right)+\frac{1}{4 \varsigma}\|d\|_{[0, t], 2}^{2}\right) \leq \underline{\psi}^{-1}\left(2 \bar{\psi}\left(\left\|\tilde{x}_{0}\right\|\right)\right)+\underline{\psi}^{-1}\left(\frac{1}{2 \varsigma}\|d\|_{[0, t], 2}^{2}\right)
$$

for every $t \in\left[0, T_{\tilde{x}_{0}, d}\right)$. So, (3.14) follows because $\underline{\sigma}, \underline{\gamma}$ defined by

$$
\underline{\sigma}(r):=\underline{\psi}^{-1}(2 \bar{\psi}(r)) \quad \text { and } \quad \underline{\gamma}(r):=\underline{\psi}^{-1}\left(\frac{1}{2 \varsigma} r^{2}\right)
$$

obviously belong to the class $\mathcal{K}$.

As a fourth and last step, we show that the maximal existence time $T_{\tilde{x}_{0}, d}=\infty$. Combined with the previous steps, this proves the theorem. So let $\left(\tilde{x}_{0}, d\right) \in \mathcal{D}$ and assume that $T_{\tilde{x}_{0}, d}<\infty$, that is, the existence interval $\left[0, T_{\tilde{x}_{0}, d}\right)$ of the maximal classical solution $\tilde{x}\left(\cdot, \tilde{x}_{0}, d\right)-\tilde{R} d$ of $(3.4)$ is bounded. It then follows by the standard blow-up result for semilinear evolution equations (Thm. 6.1 .4 of [39]) that the solution $\tilde{x}\left(\cdot, \tilde{x}_{0}, d\right)-\tilde{R} d$ of $(3.4)$ blows up. And therefore $\tilde{x}\left(\cdot, \tilde{x}_{0}, d\right)$ blows up as well:

$$
\sup _{t \in\left[0, T_{\tilde{x}_{0}, d}\right)}\left\|\tilde{x}\left(t, \tilde{x}_{0}, d\right)\right\| \geq \sup _{t \in\left[0, T_{\tilde{x}_{0}, d}\right)}\left\|\tilde{x}\left(t, \tilde{x}_{0}, d\right)-\tilde{R} d(t)\right\|-\|\tilde{R}\|\|d\|_{\left[0, T_{\tilde{x}_{0}, d}\right), \infty}=\infty
$$

(recall that $d$ is continuous with compact support). Contradiction to the estimate from the third step!

It can be shown that for $\left(\tilde{x}_{0}, d\right) \in \tilde{X} \times W_{\text {loc }}^{1,2}\left([0, \infty), \mathbb{R}^{k}\right)$, the initial value problem (3.1) still has a (unique) global mild solution, that is, a continuous function $\tilde{x}:[0, \infty) \rightarrow \tilde{X}$ satisfying the variation-of-constants formula (3.9). In order to see this - especially the global existence - one can make a density and approximation argument similar to the one from the next section: one first trivially extends $\Phi_{t}$ to a bounded linear operator on $W^{1,2}\left([0, \infty), \mathbb{R}^{k}\right)$ and then uses the same arguments as in Theorem 3.4. Since we will not need mild solutions in the sequel, however, we omit the details.

\subsection{Solvability in the generalized sense}

In this section, we show that for sufficiently irregular initial states $\tilde{x}_{0}$ and disturbances $d$, namely for

$$
\left(\tilde{x}_{0}, d\right) \in \tilde{X} \times L_{\text {loc }}^{2}\left([0, \infty), \mathbb{R}^{k}\right)
$$


(set of generalized data), the closed-loop equation (3.1) still has a generalized solution existing globally in time and arising as a suitable limit of classical solutions. In showing the existence of such limits we will make use of the integral equation

$$
\tilde{x}\left(t, \tilde{x}_{0}, d\right)=\mathrm{e}^{\tilde{A} t} \tilde{x}_{0}+\int_{0}^{t} \mathrm{e}^{\tilde{A}(t-s)} \tilde{f}\left(\tilde{x}\left(s, \tilde{x}_{0}, d\right)\right) \mathrm{d} s+\Phi_{t}(d)
$$

for classical solutions $\tilde{x}\left(\cdot, \tilde{x}_{0}, d\right)$ with $\left(\tilde{x}_{0}, d\right) \in \mathcal{D}$ from (3.21) above. In order to extend this equation to arbitrary $\left(\tilde{x}_{0}, d\right) \in \tilde{X} \times L_{\text {loc }}^{2}\left([0, \infty), \mathbb{R}^{k}\right)$, we first extend the linear operators $\Phi_{t}$ from (3.10).

Lemma 3.3. Suppose that Conditions 2.1, 2.2, 3.1 are satisfied. Then the linear operator $\Phi_{t}: C_{c}^{2}\left([0, \infty), \mathbb{R}^{k}\right) \rightarrow$ $\tilde{X}$ defined by (3.10) can be (uniquely) extended to a bounded linear operator $\bar{\Phi}_{t}: L^{2}\left([0, \infty), \mathbb{R}^{k}\right) \rightarrow \tilde{X}$ for every $t \in[0, \infty)$ and

$$
\sup _{t \in[0, \tau]}\left\|\bar{\Phi}_{t}\right\| \leq\left\|\bar{\Phi}_{\tau}\right\| \quad(\tau \in[0, \infty)) .
$$

Proof. In the entire proof, we use the short-hand notation

$$
\rho\left(\tilde{x}_{0}, d\right):=\underline{\sigma}\left(\left\|\tilde{x}_{0}\right\|\right)+\underline{\gamma}\left(\|d\|_{2}\right)
$$

for $\tilde{x}_{0} \in \tilde{X}$ and $d \in L^{2}\left([0, \infty), \mathbb{R}^{k}\right)$, where $\underline{\sigma}, \underline{\gamma} \in \mathcal{K}$ are chosen as in Theorem 3.2. We also denote by $L(\rho)$ for $\rho \in[0, \infty)$ a Lipschitz constant of $\left.\tilde{f}\right|_{\bar{B}_{\rho}(0)}$ such that $\rho \mapsto L(\rho)$ is continuous and monotonically increasing. Such a choice of Lipschitz constants exists because

$$
\rho \mapsto L_{0}(\rho):=\min \left\{L \in[0, \infty): L \text { is a Lipschitz constant of }\left.\tilde{f}\right|_{\bar{B}_{\rho}(0)}\right\}
$$

is monotonically increasing and therefore obviously has a continuous monotonically increasing majorant. (See Lem. 2.5 of [4].)

As a first step, we show that the restriction $\Phi_{t, 0}$ of $\Phi_{t}$ to

$$
C_{c, 0}^{2}\left([0, \infty), \mathbb{R}^{k}\right):=\left\{d \in C_{c}^{2}\left([0, \infty), \mathbb{R}^{k}\right): d(0)=0\right\}
$$

can be (uniquely) extended to a bounded linear operator $\bar{\Phi}_{t, 0}: L^{2}\left([0, \infty), \mathbb{R}^{k}\right) \rightarrow \tilde{X}$ for every $t \in[0, \infty)$ and that

$$
\sup _{t \in[0, \tau]}\left\|\bar{\Phi}_{t, 0}\right\| \leq\left\|\bar{\Phi}_{\tau, 0}\right\|
$$

for every $\tau \in[0, \infty)$. So let $t \in[0, \infty)$. We see by (3.21) that

$$
\Phi_{t, 0}(d)=\tilde{x}(t, 0, d)-\int_{0}^{t} \mathrm{e}^{\tilde{A}(t-s)} \tilde{f}(\tilde{x}(s, 0, d)) \mathrm{d} s
$$

for every $d \in C_{c, 0}^{2}\left([0, \infty), \mathbb{R}^{k}\right)$ (because for such $d$ one has $\left.(0, d) \in \mathcal{D}\right)$. It follows by Theorem 3.2 (ii) that

$$
\|\tilde{x}(t, 0, d)\| \leq \underline{\gamma}\left(\|d\|_{[0, t], 2}\right)
$$


for all $d \in C_{c, 0}^{2}\left([0, \infty), \mathbb{R}^{k}\right)$. It also follows by Theorem 3.2 (ii) that

$$
\left\|\int_{0}^{t} \mathrm{e}^{\tilde{A}(t-s)} \tilde{f}(\tilde{x}(s, 0, d)) \mathrm{d} s\right\| \leq L(\rho(0, d)) \underline{\gamma}\left(\|d\|_{[0, t], 2}\right) t
$$

for all $d \in C_{c, 0}^{2}\left([0, \infty), \mathbb{R}^{k}\right)$. Combining now (3.25), (3.26) and (3.27) we see that

$$
\left\|\Phi_{t, 0}(d)\right\| \leq(1+L(\rho(0, d)) t) \underline{\gamma}\left(\|d\|_{[0, t], 2}\right)
$$

for all $d \in C_{c, 0}^{2}\left([0, \infty), \mathbb{R}^{k}\right)$. It follows that

$$
\Phi_{t, 0}: C_{c, 0}^{2}\left([0, \infty), \mathbb{R}^{k}\right) \rightarrow \tilde{X}
$$

is a linear operator that is bounded w.r.t. the norm of $L^{2}\left([0, \infty), \mathbb{R}^{k}\right)$ and thus, by the density of $C_{c, 0}^{2}\left([0, \infty), \mathbb{R}^{k}\right)$ in $L^{2}\left([0, \infty), \mathbb{R}^{k}\right)$, can be uniquely extended to a bounded linear operator $\bar{\Phi}_{t, 0}: L^{2}\left([0, \infty), \mathbb{R}^{k}\right) \rightarrow \tilde{X}$. Since for every $\tau \geq t$ one has the elementary relation

$$
\Phi_{t, 0}(d)=\Phi_{\tau, 0}\left(0 \&_{\tau-t} d\right) \quad\left(d \in C_{c, 0}^{2}\left([0, \infty), \mathbb{R}^{k}\right)\right)
$$

(called composition property in [55]), it further follows that

$$
\begin{aligned}
\sup _{t \in[0, \tau]} & \left\|\bar{\Phi}_{t, 0}\right\|=\sup _{t \in[0, \tau]} \sup \left\{\left\|\Phi_{t, 0}(d)\right\|: d \in C_{c, 0}^{2}\left([0, \infty), \mathbb{R}^{k}\right) \text { with }\|d\|_{2} \leq 1\right\} \\
\leq & \sup _{t \in[0, \tau]} \sup \left\{\left\|\Phi_{\tau, 0}\left(0 \&_{\tau-t} d\right)\right\|: d \in C_{c, 0}^{2}\left([0, \infty), \mathbb{R}^{k}\right) \text { with }\|d\|_{2} \leq 1\right\} \leq\left\|\bar{\Phi}_{\tau, 0}\right\| .
\end{aligned}
$$

In the relations above, $u \&_{\tau} v$ denotes the concatenation of two functions $u, v:[0, \infty) \rightarrow \mathbb{R}^{k}$ at time $\tau$, that is, $\left(u \&_{\tau} v\right)(s):=u(s)$ for $s \in[0, \tau)$ and $\left(u \&_{\tau} v\right)(s):=v(s-\tau)$ for $s \in[\tau, \infty)$.

As a second step, we will show that also the non-restricted operator $\Phi_{t}$ can be (uniquely) extended to a bounded linear operator $\bar{\Phi}_{t}: L^{2}\left([0, \infty), \mathbb{R}^{k}\right) \rightarrow \tilde{X}$ and that $\bar{\Phi}_{t}=\bar{\Phi}_{t, 0}$ for every $t \in[0, \infty)$, which in conjunction with (3.24) proves the lemma. In order to do so, we have only to show that

$$
\bar{\Phi}_{t, 0}(d)=\Phi_{t}(d)
$$

for every $d \in C_{c}^{2}\left([0, \infty), \mathbb{R}^{k}\right)$. So let $t \in(0, \infty)$ and $d \in C_{c}^{2}\left([0, \infty), \mathbb{R}^{k}\right)$ be fixed (for $t=0$ the claim is obvious). Choose a sequence $\left(d_{n}\right)$ in $C_{c, 0}^{2}\left([0, \infty), \mathbb{R}^{k}\right)$ such that

$$
\left.d_{n}\right|_{[t / n, \infty)}=\left.d\right|_{[t / n, \infty)} \quad \text { and }\left.\quad \sup _{n \in \mathbb{N}}|| d_{n}\right|_{[0, t / n)} \|_{\infty}<\infty
$$

It then follows that $d_{n} \longrightarrow d$ in $L^{2}\left([0, \infty), \mathbb{R}^{k}\right)$. So, on the one hand

$$
\Phi_{t, 0}\left(d_{n}\right) \longrightarrow \bar{\Phi}_{t, 0}(d) \quad(n \rightarrow \infty)
$$

and on the other hand

$$
\Phi_{t, 0}\left(d_{n}\right)=\tilde{R} d_{n}(t)+e^{\tilde{A}(t-t / n)} \int_{0}^{t / n} \mathrm{e}^{\tilde{A}(t / n-s)}\left(\tilde{\mathcal{A}} \tilde{R} d_{n}(s)-\tilde{R} d_{n}^{\prime}(s)\right) \mathrm{d} s+\int_{t / n}^{t} e^{\tilde{A}(t-s)}\left(\tilde{\mathcal{A}} \tilde{R} d(s)-\tilde{R} d^{\prime}(s)\right) \mathrm{d} s
$$




$$
\begin{aligned}
& =\tilde{R} d_{n}(t)+e^{\tilde{A}(t-t / n)}\left(\Phi_{t / n, 0}\left(d_{n}\right)-\tilde{R} d_{n}(t / n)\right)+\int_{t / n}^{t} e^{\tilde{A}(t-s)}\left(\tilde{\mathcal{A}} \tilde{R} d(s)-\tilde{R} d^{\prime}(s)\right) \mathrm{d} s \\
& \longrightarrow \Phi_{t}(d) \quad(n \rightarrow \infty),
\end{aligned}
$$

where we used (3.28) to get $\Phi_{t / n, 0}\left(d_{n}\right) \longrightarrow 0$ as $n \rightarrow \infty$. Combining now (3.32) and (3.33) we finally obtain (3.30), as desired.

It should be noticed that the above lemma means nothing but the (finite-time) admissibility of the linear boundary control system

$$
\tilde{x}^{\prime}=\tilde{\mathcal{A}} \tilde{x} \quad \text { with } \quad d(t)=\tilde{\mathcal{B}} \tilde{x}(t)
$$

w.r.t. inputs $d \in L^{2}\left([0, \infty), \mathbb{R}^{k}\right)$. With the lemma at hand, we can now prove the following approximation result.

Theorem 3.4. Suppose that Conditions 2.1, 2.2, 3.1 are satisfied. Then for every $\left(\tilde{x}_{0}, d\right) \in \tilde{X} \times L_{\text {loc }}^{2}\left([0, \infty), \mathbb{R}^{k}\right)$ one has:

(i) there exists a sequence $\left(\tilde{x}_{0 n}, d_{n}\right)$ in $\mathcal{D}$ converging to $\left(\tilde{x}_{0}, d\right)$ in the locally convex topology of $\tilde{X} \times$ $L_{\mathrm{loc}}^{2}\left([0, \infty), \mathbb{R}^{k}\right)$

(ii) for every such approximating sequence $\left(\tilde{x}_{0 n}, d_{n}\right)$ in $\mathcal{D}$, the corresponding sequence $\left(\tilde{x}\left(\cdot, \tilde{x}_{0 n}, d_{n}\right)\right)$ of classical solutions of (3.1) is a Cauchy sequence in the locally convex space $C([0, \infty), \tilde{X})$ and its limit is independent of the particular choice of the approximating sequence $\left(\tilde{x}_{0 n}, d_{n}\right)$.

Proof. (i) Since $D(\tilde{A})$ is dense in $\tilde{X}$ (Lem. 2.3) and since $C_{c}^{\infty}\left((0, \infty), \mathbb{R}^{k}\right)$ is dense in $L_{\text {loc }}^{2}\left([0, \infty), \mathbb{R}^{k}\right)$, we see that

$$
D(\tilde{A}) \times C_{c}^{\infty}\left((0, \infty), \mathbb{R}^{k}\right)
$$

is dense in $\tilde{X} \times L_{\text {loc }}^{2}\left([0, \infty), \mathbb{R}^{k}\right)$. Since, moreover,

$$
D(\tilde{A}) \times C_{c}^{\infty}\left((0, \infty), \mathbb{R}^{k}\right) \subset \mathcal{D} \subset \tilde{X} \times L_{\mathrm{loc}}^{2}\left([0, \infty), \mathbb{R}^{k}\right),
$$

we see that $\mathcal{D}$ is a fortiori dense in $\tilde{X} \times L_{\text {loc }}^{2}\left([0, \infty), \mathbb{R}^{k}\right)$.

(ii) As in the proof of Lemma 3.3, choose Lipschitz constants $L(\rho)$ of $\left.\tilde{f}\right|_{\bar{B}_{\rho}(0)}$ such that $\rho \mapsto L(\rho)$ is continuous and monotonically increasing. We show that for arbitrary $\left(\tilde{x}_{01}, d_{1}\right),\left(\tilde{x}_{02}, d_{2}\right) \in \mathcal{D}$ and $\tau \in[0, \infty)$ one has the estimate

$$
\begin{array}{r}
\left\|\tilde{x}\left(\cdot, \tilde{x}_{01}, d_{1}\right)-\tilde{x}\left(\cdot, \tilde{x}_{02}, d_{2}\right)\right\|_{[0, \tau], \infty} \leq\left(\left\|\tilde{x}_{01}-\tilde{x}_{02}\right\|+\left\|\bar{\Phi}_{\tau}\right\|\left\|d_{1}-d_{2}\right\|_{[0, \tau], 2}\right) . \\
\cdot \exp \left(L\left(\rho_{\tau}\left(\tilde{x}_{01}, d_{1}, \tilde{x}_{02}, d_{2}\right)\right) \tau\right)
\end{array}
$$

where $\rho_{\tau}\left(\tilde{x}_{01}, d_{1}, \tilde{x}_{02}, d_{2}\right):=\rho_{\tau}\left(\tilde{x}_{01}, d_{1}\right)+\rho_{\tau}\left(\tilde{x}_{02}, d_{2}\right)$ and

$$
\rho_{\tau}\left(\tilde{x}_{0}, d\right):=\underline{\sigma}\left(\left\|\tilde{x}_{0}\right\|\right)+\underline{\gamma}\left(\|d\|_{[0, \tau], 2}\right)
$$

for $\left(\tilde{x}_{0}, d\right) \in \tilde{X} \times L_{\text {loc }}^{2}\left([0, \infty), \mathbb{R}^{k}\right)$ with $\underline{\sigma}, \underline{\gamma}$ as in Theorem 3.2. So let $\left(\tilde{x}_{01}, d_{1}\right),\left(\tilde{x}_{02}, d_{2}\right) \in \mathcal{D}$ and $\tau \in[0, \infty)$. It then follows from (3.21) using Lemma 2.3, Theorem 3.2 and Lemma 3.3 that

$$
\left\|\tilde{x}\left(t, \tilde{x}_{01}, d_{1}\right)-\tilde{x}\left(t, \tilde{x}_{02}, d_{2}\right)\right\| \leq\left\|\tilde{x}_{01}-\tilde{x}_{02}\right\|+\left\|\bar{\Phi}_{\tau}\right\|\left\|d_{1}-d_{2}\right\|_{[0, \tau], 2}
$$




$$
+L\left(\rho_{\tau}\left(\tilde{x}_{01}, d_{1}, \tilde{x}_{02}, d_{2}\right)\right) \int_{0}^{t}\left\|\tilde{x}\left(s, \tilde{x}_{01}, d_{1}\right)-\tilde{x}\left(s, \tilde{x}_{02}, d_{2}\right)\right\| \mathrm{d} s
$$

for every $t \in[0, \tau]$. So, by Grönwall's lemma, the claimed estimate (3.35) follows. And from this, in turn, assertion (ii) immediately follows because $\left(\tilde{X} \times L_{\text {loc }}^{2}\left([0, \infty), \mathbb{R}^{k}\right)\right)^{2} \ni\left(\tilde{x}_{01}, d_{1}, \tilde{x}_{02}, d_{2}\right) \mapsto \rho_{\tau}\left(\tilde{x}_{01}, d_{1}, \tilde{x}_{02}, d_{2}\right)$ is continuous.

As a consequence of the above theorem, for every generalized datum $\left(\tilde{x}_{0}, d\right) \in \tilde{X} \times L_{\text {loc }}^{2}\left([0, \infty), \mathbb{R}^{k}\right)$, the assignment

$$
\tilde{x}\left(\cdot, \tilde{x}_{0}, d\right):=\lim _{n \rightarrow \infty} \tilde{x}\left(\cdot, \tilde{x}_{0 n}, d_{n}\right) \in C([0, \infty), \tilde{X})
$$

with $\left(\tilde{x}_{0 n}, d_{n}\right)$ being an arbitrary approximating sequence in $\mathcal{D}$ for $\left(\tilde{x}_{0}, d\right)$, yields a well-defined function. We call this function - following [52] - the generalized solution of the closed-loop system (3.1) corresponding to $\left(\tilde{x}_{0}, d\right)$ because it obviously coincides with the classical solution for $\left(\tilde{x}_{0}, d\right) \in \mathcal{D}$ (and with the mild solution for $\left.\left(\tilde{x}_{0}, d\right) \in \tilde{X} \times W_{\text {loc }}^{1,2}\left([0, \infty), \mathbb{R}^{k}\right)\right)$ and because, by the next corollary, it shares many important properties with classical solutions. In particular, the generalized solutions of our closed-loop system satisfy the axioms from [30] (Def. 1).

Corollary 3.5. Suppose that Conditions 2.1, 3.1, 2.2 are satisfied. Then

(i) the generalized solution map $\left(\tilde{x}_{0}, d\right) \mapsto \tilde{x}\left(\cdot, \tilde{x}_{0}, d\right)$ satisfies the cocycle (or flow) property, that is,

$$
\tilde{x}\left(t+s, \tilde{x}_{0}, d\right)=\tilde{x}\left(t, \tilde{x}\left(s, \tilde{x}_{0}, d\right), d(s+\cdot)\right)
$$

for all $s, t \in[0, \infty)$ and all $\left(\tilde{x}_{0}, d\right) \in \tilde{X} \times L_{\mathrm{loc}}^{2}\left([0, \infty), \mathbb{R}^{k}\right)$

(ii) the generalized solution map $\left(\tilde{x}_{0}, d\right) \mapsto \tilde{x}\left(\cdot, \tilde{x}_{0}, d\right) \in C([0, \infty), \tilde{X})$ is continuous and causal.

Proof. Assertion (i) and the causality part of assertion (ii) easily follow by approximation from the cocycle property and the causality of classical solutions. Similarly, the continuity part of assertion (ii) follows by extending the estimate (3.35) to generalized solutions.

It can be shown that the closed-loop system, in addition to having a generalized solution, also has a generalized output $y\left(\cdot, \tilde{x}_{0}, d\right) \in L_{\text {loc }}^{2}\left([0, \infty), \mathbb{R}^{k}\right)$ for every $\left(\tilde{x}_{0}, d\right) \in \tilde{X} \times L_{\text {loc }}^{2}\left([0, \infty), \mathbb{R}^{k}\right)$ and that the generalized output map

$$
\tilde{X} \times L_{\mathrm{loc}}^{2}\left([0, \infty), \mathbb{R}^{k}\right) \ni\left(\tilde{x}_{0}, d\right) \mapsto y\left(\cdot, \tilde{x}_{0}, d\right) \in L_{\mathrm{loc}}^{2}\left([0, \infty), \mathbb{R}^{k}\right)
$$

is continuous in the respective locally convex topology. In particular, the closed-loop system is well-posed in the spirit of [52] - but we will not need this in the sequel. See [46, 47] for proofs and general well-posedness results.

\section{Stability of THE CLOSED-LOOP SYSTEM}

After having established the global solvability of the closed-loop system, we can now move on to stability. A first very simple result is the following uniform global stability theorem. We recall from [30] that the system $\tilde{\mathfrak{S}}$ is called uniformly globally stable iff there exist comparison functions $\underline{\sigma}, \underline{\gamma} \in \mathcal{K}$ such that for every $\tilde{x}_{0} \in \tilde{X}$ and $d \in L^{2}\left([0, \infty), \mathbb{R}^{k}\right)$

$$
\left\|\tilde{x}\left(t, \tilde{x}_{0}, d\right)\right\| \leq \underline{\sigma}\left(\left\|\tilde{x}_{0}\right\|\right)+\underline{\gamma}\left(\|d\|_{2}\right) \quad(t \in[0, \infty)) .
$$

Theorem 4.1. Suppose that the assumptions (Conditions 2.1, 2.2, 3.1) of the solvability theorems are satisfied. Then the closed-loop system $\tilde{\mathfrak{S}}$ is uniformly globally stable. 
Proof. An immediate consequence of Theorem 3.2 (ii) and (3.38).

We are now going to improve this result to a (uniform) input-to-state stability result (Sect. 4.1) and to a weak input-to-state stability result (Sect. 4.2), respectively. In particular, we prove that the generalized solutions $\tilde{x}\left(t, \tilde{x}_{0}, d\right)$ of the closed-loop system converge to 0 as $t \rightarrow \infty$ for every initial state $\tilde{x}_{0}$ and every square-integrable disturbance $d$. In the case of finite-dimensional systems, such a convergence result has been proved in [20] under a strict output passivity and a zero-state detectability assumption. We point out, however, that the theory from [20] cannot be applied in our situation. Indeed, the finite-dimensionality of the systems considered in [20] is essentially used in the proof from [20] (namely to get the relative compactness of orbits needed for the invariance principle).

\subsection{Input-to-state stability of the closed-loop system}

In this section we show that, for systems $\mathfrak{S}$ of order $N=1$ and for a special class of controllers $\mathfrak{S}_{c}$, the closed-loop system $\tilde{\mathfrak{S}}$ is (uniformly) input-to-state stable. We recall that $\tilde{\mathfrak{S}}$ is (uniformly) input-to-state stable w.r.t. inputs from $L^{2}\left([0, \infty), \mathbb{R}^{k}\right)$ iff it is uniformly globally stable and of uniform asymptotic gain. See [30] for this and other characterizations of input-to-state stability. In this context, the uniform asymptotic gain property by definition means the following: there is a function $\bar{\gamma} \in \mathcal{K} \cup\{0\}$ such that for every $\varepsilon, r>0$ there is a time $\bar{\tau}=\bar{\tau}(\varepsilon, r)$ such that for every $\tilde{x}_{0} \in \tilde{X}$ with $\left\|\tilde{x}_{0}\right\| \leq r$ and every $d \in L^{2}\left([0, \infty), \mathbb{R}^{k}\right)$

$$
\left\|\tilde{x}\left(t, \tilde{x}_{0}, d\right)\right\| \leq \varepsilon+\bar{\gamma}\left(\|d\|_{2}\right) \quad(t \geq \bar{\tau}) .
$$

A function $\bar{\gamma}$ as above is called a uniform (asymptotic) gain (function) for $\tilde{\mathfrak{S}}$.

Lemma 4.2. If the assumptions (Conditions 2.1, 2.2, 3.1) of the solvability results are satisfied and $\tilde{\mathfrak{S}}$ is uniformly input-to-state stable w.r.t. inputs from $L^{2}\left([0, \infty), \mathbb{R}^{k}\right)$, then for every $\left(\tilde{x}_{0}, d\right) \in \tilde{X} \times L^{2}\left([0, \infty), \mathbb{R}^{k}\right)$ one has

$$
\tilde{x}\left(t, \tilde{x}_{0}, d\right) \longrightarrow 0 \quad(t \rightarrow \infty)
$$

Proof. Choose $\varepsilon>0$ and $\left(\tilde{x}_{0}, d\right) \in \tilde{X} \times L^{2}\left([0, \infty), \mathbb{R}^{k}\right)$. Since $\tilde{\mathfrak{S}}$ is uniformly globally stable and of uniform asymptotic gain, there exist $\underline{\sigma}, \underline{\gamma} \in \mathcal{K}$ as in (4.1) and a uniform gain function $\bar{\gamma} \in \mathcal{K} \cup\{0\}$ along with a time $\bar{\tau}_{0}=\bar{\tau}_{0}\left(\varepsilon, \underline{\sigma}\left(\left\|\tilde{x}_{0}\right\|\right)+\underline{\gamma}\left(\|d\|_{2}\right)\right)$ such that

$$
\left\|\tilde{x}\left(t, \tilde{\xi}_{0}, d\right)\right\| \leq \varepsilon+\bar{\gamma}\left(\|d\|_{2}\right)
$$

for every $\tilde{\xi}_{0} \in \tilde{X}$ with $\left\|\tilde{\xi}_{0}\right\| \leq \underline{\sigma}\left(\left\|\tilde{x}_{0}\right\|\right)+\underline{\gamma}\left(\|d\|_{2}\right)$ and every $t \geq \bar{\tau}_{0}$. Since moreover $d \in L^{2}\left([0, \infty), \mathbb{R}^{k}\right)$, there exists a time $t_{0}=t_{0}(\varepsilon, d)$ such that

$$
\bar{\gamma}\left(\left\|d\left(t_{0}+\cdot\right)\right\|_{2}\right)<\varepsilon
$$

Setting now $\bar{\tau}:=\bar{\tau}\left(\varepsilon,\left\|\tilde{x}_{0}\right\|, d\right):=\bar{\tau}_{0}+t_{0}$, we see by the cocycle property and by (4.1), (4.4), (4.5) that $\left\|\tilde{x}\left(t^{\prime}, \tilde{x}_{0}, d\right)\right\|<2 \varepsilon$ for all $t^{\prime} \geq \bar{\tau}$. And so (4.3) ensues.

In order to achieve input-to-state stability, we add the following conditions on the system $\mathfrak{S}$ and the controller $\mathfrak{S}_{c}$ to the assumptions from the solvability results.

Condition 4.3. $\mathfrak{S}$ is of order $N=1$ and $\zeta \mapsto \mathcal{H}(\zeta)$ is absolutely continuous. Additionally, there exists a constant $\kappa>0$ such that for $\eta=a$ or $\eta=b$ one has

$$
|\mathcal{B} x|^{2}+|\mathcal{C} x|^{2} \geq \kappa|(\mathcal{H} x)(\eta)|^{2} \quad(x \in D(\mathcal{A})) .
$$


Condition 4.4. $\quad$ (i) $\mathcal{P}$ is quasi-quadratic in the sense that for some constants $\underline{c}_{1}, \bar{c}_{1}>0$

$$
\bar{c}_{1} v_{1}^{\top} \nabla \mathcal{P}\left(v_{1}\right) \geq \mathcal{P}\left(v_{1}\right) \geq \underline{c}_{1}\left|v_{1}\right|^{2} \quad\left(v_{1} \in \mathbb{R}^{m_{c}}\right)
$$

(ii) $\mathcal{R}$ is quasi-linear in the sense that for some constants $\underline{c}_{2}, \bar{c}_{2}>0$

$$
\bar{c}_{2} v_{2}^{\top} \mathcal{R}\left(v_{2}\right) \geq\left|v_{2}\right|^{2} \geq \underline{c}_{2}\left|\mathcal{R}\left(v_{2}\right)\right|^{2} \quad\left(v_{2} \in \mathbb{R}^{m_{c}}\right) .
$$

Condition 4.3 can be viewed as an approximate observability condition on our system $\mathfrak{S}$ by virtue of Lemma 4.16 below.

Theorem 4.5. Suppose that the assumptions (Conditions 2.1, 2.2, 3.1) of the solvability theorems are satisfied along with Conditions 4.3 and 4.4. Then the closed-loop system $\tilde{\mathfrak{S}}$ is input-to-state stable and the function $\bar{\gamma}$, given by $\bar{\gamma}(r)=\underline{\psi}^{-1}\left(2 C r^{2}\right)$ with arbitrary $C>1 /(4 \varsigma)$ and with $\varsigma, \underline{\psi}$ as in (2.15) and (2.18), is a uniform asymptotic gain for $\tilde{\mathfrak{S}}$. In particular,

$$
\tilde{x}\left(t, \tilde{x}_{0}, d\right) \longrightarrow 0 \quad(t \rightarrow \infty)
$$

for every $\tilde{x}_{0} \in \tilde{X}$ and $d \in L^{2}\left([0, \infty), \mathbb{R}^{k}\right)$.

We now turn to the proof of the theorem and we begin by recording some central ingredients.

\subsubsection{Central ingredients of the proof}

A first important ingredient is the following estimate for the energy along solutions which essentially uses our assumption that $\tilde{\mathfrak{S}}$ be of order $N=1$. It is a perturbed version of the respective sideways energy estimate from [42].

Lemma 4.6. Under the assumptions of the above theorem, there exists a function $c_{0} \in \mathcal{L}$ and a $t_{0}>0$ such that for $\eta=a$ and $\eta=b$ one has

$$
\tilde{E}\left(\tilde{x}\left(t, \tilde{x}_{0}, d\right)\right) \leq c_{0}(t)\left(\int_{0}^{t}|(\mathcal{H} x(s))(\eta)|^{2} \mathrm{~d} s+\int_{0}^{t} E_{c}(v(s)) \mathrm{d} s\right)+\int_{0}^{t}|d(s)||y(s)| \mathrm{d} s
$$

for every $\left(\tilde{x}_{0}, d\right) \in \mathcal{D}$ and for every $t \geq t_{0}$, where $(x, v):=\tilde{x}\left(\cdot, \tilde{x}_{0}, d\right)$ and $y:=y\left(\cdot, \tilde{x}_{0}, d\right)$.

Proof. As a first step, we show that there exist constants $\gamma_{0}, \kappa_{0} \in(0, \infty)$ such that for every $\left(\tilde{x}_{0}, d\right) \in \mathcal{D}$ one has

$$
F_{\tau}^{+}(\zeta) \leq F_{\tau}^{+}(b) \mathrm{e}^{\kappa_{0}(b-a)} \quad \text { and } \quad F_{\tau}^{-}(\zeta) \leq F_{\tau}^{-}(a) \mathrm{e}^{\kappa_{0}(b-a)}
$$

for every $\zeta \in(a, b)$ and every $\tau>2 \gamma_{0}(b-a)$, where

$$
\begin{aligned}
& F_{\tau}^{+}(\zeta):=F_{\tau,\left(\tilde{x}_{0}, d\right)}^{+}(\zeta):=\int_{\gamma_{0}(b-\zeta)}^{\tau-\gamma_{0}(b-\zeta)} x(s, \zeta)^{\top} \mathcal{H}(\zeta) x(s, \zeta) \mathrm{d} s \\
& F_{\tau}^{-}(\zeta):=F_{\tau,\left(\tilde{x}_{0}, d\right)}^{-}(\zeta):=\int_{\gamma_{0}(\zeta-a)}^{\tau-\gamma_{0}(\zeta-a)} x(s, \zeta)^{\top} \mathcal{H}(\zeta) x(s, \zeta) \mathrm{d} s
\end{aligned}
$$

and where $x(s, \cdot)$ denotes the continuous representative of $x(s)$ and, as usual, $(x, v):=\tilde{x}\left(\cdot, \tilde{x}_{0}, d\right)$. Indeed, set

$$
\gamma_{0}:=\left\|P_{1}^{-1}\right\| / \underline{m} \quad \text { and } \quad \kappa_{0}:=\left(2\left\|P_{1}^{-1} P_{0}\right\| \bar{m}+\bar{m}^{\prime}\right) / \underline{m}
$$


where $\underline{m}, \bar{m}$ are as in $(2.4)$ and $\bar{m}^{\prime}:=\left(\int_{a}^{b}\left\|\mathcal{H}^{\prime}(\theta)\right\| \mathrm{d} \theta\right) /(b-a)$. Also, choose and fix $\left(\tilde{x}_{0}, d\right) \in \mathcal{D}$. Since $x$ is a classical solution of

$$
x^{\prime}=\mathcal{A} x,
$$

and $\mathcal{H}$ is absolutely continuous, it follows by Lemma 3.2 of [44] that $F_{\tau}^{ \pm}$for every $\tau>2 \gamma_{0}(b-a)$ is absolutely continuous and hence differentiable almost everywhere with derivative given by

$$
\begin{aligned}
\left(F_{\tau}^{ \pm}\right)^{\prime}(\zeta)=x & x, \zeta)\left.^{\top}\left( \pm \gamma_{0} \mathcal{H}(\zeta)+P_{1}^{-1}\right) x(s, \zeta)\right|_{s=t^{ \pm}(\zeta)}+\left.x(s, \zeta)^{\top}\left( \pm \gamma_{0} \mathcal{H}(\zeta)-P_{1}^{-1}\right) x(s, \zeta)\right|_{s=r^{ \pm}(\zeta)} \\
& -\int_{r^{ \pm}(\zeta)}^{t^{ \pm}(\zeta)} x(s, \zeta)^{\top}\left(\left(P_{1}^{-1} P_{0} \mathcal{H}(\zeta)\right)^{\top}+\mathcal{H}^{\prime}(\zeta)+P_{1}^{-1} P_{0} \mathcal{H}(\zeta)\right) x(s, \zeta) \mathrm{d} s
\end{aligned}
$$

for a.e. $\zeta \in(a, b)$, where $r^{+}(\zeta):=\gamma_{0}(b-\zeta), t^{+}(\zeta):=\tau-\gamma_{0}(b-\zeta)$ and $r^{-}(\zeta):=\gamma_{0}(\zeta-a), t^{-}(\zeta):=\tau-\gamma_{0}(\zeta-a)$. In view of (4.9) it follows from (4.10) that

$$
\begin{aligned}
& \left(F_{\tau}^{+}\right)^{\prime}(\zeta) \geq-\kappa(\zeta) \int_{r^{+}(\zeta)}^{t^{+}(\zeta)} x(s, \zeta)^{\top} \underline{m} x(s, \zeta) \mathrm{d} s \geq-\kappa(\zeta) F_{\tau}^{+}(\zeta) \\
& \left(F_{\tau}^{-}\right)^{\prime}(\zeta) \leq \kappa(\zeta) \int_{r^{-}(\zeta)}^{t^{-}(\zeta)} x(s, \zeta)^{\top} \underline{m} x(s, \zeta) \mathrm{d} s \leq \kappa(\zeta) F_{\tau}^{-}(\zeta)
\end{aligned}
$$

for all $\tau>2 \gamma_{0}(b-a)$ and a.e. $\zeta \in(a, b)$, where

$$
\kappa(\zeta):=\left(2\left\|P_{1}^{-1} P_{0}\right\| \bar{m}+\left\|\mathcal{H}^{\prime}(\zeta)\right\|\right) / \underline{m} .
$$

Since $F_{\tau}^{ \pm}$is absolutely continuous, the differential inequalities (4.11) and (4.12) imply that

$$
\zeta \mapsto F_{\tau}^{+}(\zeta) \exp \left(-\int_{\zeta}^{b} \kappa(\theta) \mathrm{d} \theta\right) \quad \text { and } \quad \zeta \mapsto F_{\tau}^{-}(\zeta) \exp \left(-\int_{a}^{\zeta} \kappa(\theta) \mathrm{d} \theta\right)
$$

are monotonically increasing or decreasing, respectively. And from this, in turn, (4.8) follows in a straightforward manner.

As a second step, we show the assertion of the lemma. Choose $\gamma_{0}, \kappa_{0}$ as in $(4.9)$ and let $\left(\tilde{x}_{0}, d\right) \in \mathcal{D}$. In view of the first line of (3.16) we see that

$$
\tilde{E}_{\tilde{x}}\left(t_{2}\right) \leq \tilde{E}_{\tilde{x}}\left(t_{1}\right)+\int_{t_{1}}^{t_{2}}|d(s)||y(s)| \mathrm{d} s
$$

for all $t_{1} \leq t_{2}$. It follows from (4.13) that, for every $\tau>2 \gamma_{0}(b-a)$,

$$
\begin{aligned}
\left(\tau-2 \gamma_{0}(b-a)\right) \tilde{E}_{\tilde{x}}\left(\tau-\gamma_{0}(b-a)\right)=\int_{\gamma_{0}(b-a)}^{\tau-\gamma_{0}(b-a)} \tilde{E}_{\tilde{x}}\left(\tau-\gamma_{0}(b-a)\right) \mathrm{d} t \\
\quad \leq \int_{\gamma_{0}(b-a)}^{\tau-\gamma_{0}(b-a)}\left(\tilde{E}_{\tilde{x}}(t)+\int_{t}^{\tau-\gamma_{0}(b-a)}|d(s)||y(s)| \mathrm{d} s\right) \mathrm{d} t \\
\quad=\frac{1}{2} \int_{a}^{b} \int_{\gamma_{0}(b-a)}^{\tau-\gamma_{0}(b-a)} x(t, \zeta)^{\top} \mathcal{H}(\zeta) x(t, \zeta) \mathrm{d} t \mathrm{~d} \zeta+\int_{\gamma_{0}(b-a)}^{\tau-\gamma_{0}(b-a)} E_{c}(v(t)) \mathrm{d} t
\end{aligned}
$$




$$
+\int_{\gamma_{0}(b-a)}^{\tau-\gamma_{0}(b-a)} \int_{t}^{\tau-\gamma_{0}(b-a)}|d(s)||y(s)| \mathrm{d} s \mathrm{~d} t
$$

and therefore (increase the intervals of integration!)

$$
\begin{aligned}
\left(\tau-2 \gamma_{0}(b-a)\right) \tilde{E}_{\tilde{x}}\left(\tau-\gamma_{0}(b-a)\right) \leq & \frac{1}{2} \int_{a}^{b} F_{\tau}^{ \pm}(\zeta) \mathrm{d} \zeta+\int_{0}^{\tau} E_{c}(v(t)) \mathrm{d} t \\
& +\left(\tau-2 \gamma_{0}(b-a)\right) \int_{\gamma_{0}(b-a)}^{\tau-\gamma_{0}(b-a)}|d(s)||y(s)| \mathrm{d} s .
\end{aligned}
$$

It further follows from (4.13) that, for every $\tau>2 \gamma_{0}(b-a)$,

$$
\tilde{E}_{\tilde{x}}(\tau) \leq \tilde{E}_{\tilde{x}}\left(\tau-\gamma_{0}(b-a)\right)+\int_{\tau-\gamma_{0}(b-a)}^{\tau}|d(s)||y(s)| \mathrm{d} s .
$$

Combining (4.15) with (4.14) and (4.8), we conclude that

$$
\begin{aligned}
\tilde{E}\left(\tilde{x}\left(\tau, \tilde{x}_{0}, d\right)\right) \leq & \frac{1}{2\left(\tau-2 \gamma_{0}(b-a)\right)} \min \left\{F_{\tau}^{+}(b), F_{\tau}^{-}(a)\right\} \mathrm{e}^{\kappa_{0}(b-a)}(b-a) \\
& +\frac{1}{\tau-2 \gamma_{0}(b-a)} \int_{0}^{\tau} E_{c}(v(t)) \mathrm{d} t+\int_{0}^{\tau}|d(s)||y(s)| \mathrm{d} s
\end{aligned}
$$

for every $\tau>2 \gamma_{0}(b-a)$. Choosing now $t_{0}:=2 \gamma_{0}(b-a)+1$ and $c_{0} \in \mathcal{L}$ such that

$$
c_{0}(\tau)=\frac{1}{\tau-2 \gamma_{0}(b-a)} \max \left\{\mathrm{e}^{\kappa_{0}(b-a)}(b-a) /(2 \underline{m}), 1\right\}
$$

for all $\tau \geq t_{0}$, we obtain the assertion of the lemma from (4.16).

A second important ingredient is the following estimate for the integrated controller energy which shows up in the previous lemma.

Lemma 4.7. Under the assumptions of the above theorem, there exists a constant $C_{0}>0$ such that for every $\left(\tilde{x}_{0}, d\right) \in \mathcal{D}$

$$
\int_{0}^{t} E_{c}(v(s)) \mathrm{d} s \leq C_{0}\left(\tilde{E}\left(\tilde{x}_{0}\right)+\int_{0}^{t}|y(s)|^{2} \mathrm{~d} s\right)
$$

for all $t \in[0, \infty)$, where $(x, v):=\tilde{x}\left(\cdot, \tilde{x}_{0}, d\right)$ and $y:=y\left(\cdot, \tilde{x}_{0}, d\right)$.

Proof. As a first step, we show that $E_{c}$ is equivalent to the auxiliary functions $V_{\gamma}$ defined by

$$
V_{\gamma}(v):=E_{c}(v)+\gamma v_{1}^{\top} v_{2} \quad\left(v=\left(v_{1}, v_{2}\right) \in \mathbb{R}^{2 m_{c}}\right)
$$

for all sufficiently small $\gamma>0$. In fact, we show that there is a $\gamma_{0}>0$ such that for all $\gamma \in\left(0, \gamma_{0}\right]$ and $v \in \mathbb{R}^{2 m_{c}}$ one has

$$
\frac{1}{2} V_{\gamma}(v) \leq E_{c}(v) \leq 2 V_{\gamma}(v)
$$


In essence, this is Lemma 16 of [42], but in contrast to that lemma our equivalence constants in (4.18) are independent of $\gamma_{0}$ and our proof is a bit more direct. In order to see (4.18), just observe that by Condition 4.4 one has for all $v \in \mathbb{R}^{2 m_{c}}$

$$
\left|v_{1}^{\top} v_{2}\right| \leq \frac{\left|v_{1}\right|^{2}}{2}+\frac{\left|v_{2}\right|^{2}}{2} \leq \underline{c}_{1}^{-1} \mathcal{P}\left(v_{1}\right)+\left\|K^{-1}\right\| \frac{v_{2}^{\top} K v_{2}}{2} \leq C_{1} E_{c}(v)
$$

for $C_{1}:=\max \left\{\underline{c}_{1}^{-1},\left\|K^{-1}\right\|\right\}$. So, with $\gamma_{0}:=1 /\left(2 C_{1}\right)$ the equivalence relation (4.18) follows.

As a second step, we observe that there exists a constant $C_{0}>0$ and a $\gamma \in\left(0, \gamma_{0}\right]$ such that for every $\left(\tilde{x}_{0}, d\right) \in \mathcal{D}$ one has

$$
\int_{0}^{t} V_{\gamma}(v(s)) \mathrm{d} s \leq C_{0}\left(V_{\gamma}\left(v_{0}\right)+\int_{0}^{t}|y(s)|^{2} \mathrm{~d} s\right)
$$

for all $t \in[0, \infty)$, where $(x, v):=\tilde{x}\left(\cdot, \tilde{x}_{0}, d\right)$ and $y:=y\left(\cdot, \tilde{x}_{0}, d\right)$. Since this follows in the same way as Lemma 17 of [42], we omit the proof. In conjunction with (4.18), the estimate (4.20) proves the lemma.

\subsubsection{Conclusion of the proof}

With the above lemmas at hand, we can now conclude the proof of our input-to-state stability result in two steps. Since we already know that the closed-loop system is uniformly globally stable (Thm. 4.1), we have only to show that it is of uniform asymptotic gain $\bar{\gamma}:=\psi^{-1}\left(2 C{ }^{2}\right)$ for every $C>1 /(4 \varsigma)$.

As a first step, we show that for every $C>1 /(\overline{4} \varsigma)$ there exist a constant $\beta \in(0,1)$ and a time $\tau \in(0, \infty)$ such that for every $\left(\tilde{x}_{0}, d\right) \in \mathcal{D}$

$$
\tilde{E}\left(\tilde{x}\left(\tau, \tilde{x}_{0}, d\right)\right) \leq \beta \tilde{E}\left(\tilde{x}_{0}\right)+C\|d\|_{[0, \tau], 2}^{2} .
$$

So let $C>1 /(4 \varsigma)$ and let $\eta$ be the endpoint of $(a, b)$ for which (4.6) is satisfied. Also, let $\left(\tilde{x}_{0}, d\right) \in \mathcal{D}$ and, as usual, write $(x, v):=\tilde{x}\left(\cdot, \tilde{x}_{0}, d\right)$ and $u:=\mathcal{B} x, y:=\mathcal{C} x$. We know from the first line of (3.16) that

$$
\tilde{E}_{\tilde{x}}(t) \leq \tilde{E}\left(\tilde{x}_{0}\right)+\int_{0}^{t}|d(s)||y(s)| \mathrm{d} s-\bar{c}_{2}^{-1} \int_{0}^{t}\left|K v_{2}(s)\right|^{2} \mathrm{~d} s-\varsigma(1-\varepsilon) \int_{0}^{t}|y(s)|^{2} \mathrm{~d} s-\varsigma \varepsilon \int_{0}^{t}|y(s)|^{2} \mathrm{~d} s
$$

for all $t \in[0, \infty)$ and $\varepsilon \in(0,1)$, where Condition 4.4 and (2.15) have been used. Also, from (4.6) it follows that

$$
\begin{aligned}
-|y(s)|^{2} & \leq-\kappa|(\mathcal{H} x(s))(\eta)|^{2}+|u(s)|^{2}=-\kappa|(\mathcal{H} x(s))(\eta)|^{2}+\left|d(s)-B_{c}^{\top} K v_{2}(s)-S_{c} y(s)\right|^{2} \\
& \leq-\kappa|(\mathcal{H} x(s))(\eta)|^{2}+4|d(s)|^{2}+4\left\|B_{c}\right\|^{2}\left|K v_{2}(s)\right|^{2}+4\left\|S_{c}\right\|^{2}|y(s)|^{2}
\end{aligned}
$$

for all $s \in[0, \infty)$. And from Lemma 4.6 and 4.7 it follows that

$$
-\int_{0}^{t}|(\mathcal{H} x(s))(\eta)|^{2} \mathrm{~d} s \leq-\frac{1}{c_{0}(t)} \tilde{E}_{\tilde{x}}(t)+C_{0} \tilde{E}\left(\tilde{x}_{0}\right)+C_{0} \int_{0}^{t}|y(s)|^{2} \mathrm{~d} s+\frac{1}{c_{0}(t)} \int_{0}^{t}|d(s)||y(s)| \mathrm{d} s
$$

for all $t \in\left[t_{0}, \infty\right)$, where $C_{0}, t_{0}, c_{0}$ are the constants and the function from the above lemmas. Inserting now (4.23) and (4.24) in the last integral on the right-hand side of (4.22), we obtain

$$
\begin{aligned}
\tilde{E}_{\tilde{x}}(t) \leq & \tilde{E}\left(\tilde{x}_{0}\right)+\int_{0}^{t}|d(s)||y(s)| \mathrm{d} s-\bar{c}_{2}^{-1} \int_{0}^{t}\left|K v_{2}(s)\right|^{2} \mathrm{~d} s-\varsigma(1-\varepsilon) \int_{0}^{t}|y(s)|^{2} \mathrm{~d} s \\
& -\frac{\varsigma \varepsilon \kappa}{c_{0}(t)} \tilde{E}_{\tilde{x}}(t)+\varsigma \varepsilon \kappa C_{0} \tilde{E}\left(\tilde{x}_{0}\right)+\varsigma \varepsilon \kappa C_{0} \int_{0}^{t}|y(s)|^{2} \mathrm{~d} s+\frac{\varsigma \varepsilon \kappa}{c_{0}(t)} \int_{0}^{t}|d(s)||y(s)| \mathrm{d} s
\end{aligned}
$$




$$
+4 \varsigma \varepsilon \int_{0}^{t}|d(s)|^{2} \mathrm{~d} s+4 \varsigma \varepsilon\left\|B_{c}\right\|^{2} \int_{0}^{t}\left|K v_{2}(s)\right|^{2} \mathrm{~d} s+4 \varsigma \varepsilon\left\|S_{c}\right\|^{2} \int_{0}^{t}|y(s)|^{2} \mathrm{~d} s
$$

for all $t \in\left[t_{0}, \infty\right)$. So, by regrouping terms, we see that

$$
\begin{aligned}
\left(1+\frac{\varsigma \varepsilon \kappa}{c_{0}(t)}\right) \tilde{E}_{\tilde{x}}(t) \leq & \left(1+\varsigma \varepsilon \kappa C_{0}\right) \tilde{E}\left(\tilde{x}_{0}\right)+\left(4 \varsigma \varepsilon\left\|B_{c}\right\|^{2}-\bar{c}_{2}^{-1}\right) \int_{0}^{t}\left|K v_{2}(s)\right|^{2} \mathrm{~d} s \\
& +\left(\left(1+\frac{\varsigma \varepsilon \kappa}{c_{0}(t)}\right) \frac{1}{2 \alpha}+\varsigma \varepsilon \kappa C_{0}+4 \varsigma \varepsilon\left\|S_{c}\right\|^{2}-\varsigma(1-\varepsilon)\right) \int_{0}^{t}|y(s)|^{2} \mathrm{~d} s \\
& +\left(\left(1+\frac{\varsigma \varepsilon \kappa}{c_{0}(t)}\right) \frac{\alpha}{2}+4 \varsigma \varepsilon\right) \int_{0}^{t}|d(s)|^{2} \mathrm{~d} s
\end{aligned}
$$

for all $t \in\left[t_{0}, \infty\right)$ and arbitrary $\alpha \in(0, \infty)$. In particular, this holds true for $\alpha$ chosen such that

$$
\frac{1}{2 \varsigma}<\alpha<2 C
$$

(which choice is possible because $C>1 /(4 \varsigma)$ ). We choose now $\tau \in\left[t_{0}, \infty\right)$ so large that

$$
\frac{1}{c_{0}(\tau)}>C_{0}
$$

which is possible because $c_{0} \in \mathcal{L}$. We also choose $\varepsilon \in(0,1)$ so small that

$$
\begin{gathered}
4 \varsigma \varepsilon\left\|B_{c}\right\|^{2} \leq \bar{c}_{2}^{-1} \quad \text { and } \quad \frac{\alpha}{2}+\frac{4 \varsigma \varepsilon}{1+\frac{\varsigma \varepsilon \kappa}{c_{0}(\tau)}} \leq C \\
\frac{1}{2 \alpha}+\varsigma \varepsilon\left(\frac{\kappa}{c_{0}(\tau)} \frac{1}{2 \alpha}+\kappa C_{0}+4\left\|S_{c}\right\|^{2}+1\right) \leq \varsigma
\end{gathered}
$$

which last two choices are possible because of (4.26). Combining now (4.25) with (4.28), we conclude that

$$
\tilde{E}\left(\tilde{x}\left(\tau, \tilde{x}_{0}, d\right)\right) \leq \beta \tilde{E}\left(\tilde{x}_{0}\right)+C\|d\|_{[0, \tau], 2}^{2}
$$

where by virtue of (4.27) the constant $\beta$ is smaller than 1 , as desired:

$$
\beta:=\frac{1+\varsigma \varepsilon \kappa C_{0}}{1+\frac{\varsigma \kappa \kappa}{c_{0}(\tau)}}<1
$$

As a second step, we show that for every $C>1 /(4 \varsigma)$ and for every constant $\beta$ and time $\tau$ as in the first step one has

$$
\tilde{E}\left(\tilde{x}\left(t, \tilde{x}_{0}, d\right)\right) \leq \frac{1}{\beta} \beta^{t / \tau} \tilde{E}\left(\tilde{x}_{0}\right)+C\|d\|_{[0, t], 2}^{2}
$$

for every $t \in[0, \infty)$ and every $\left(\tilde{x}_{0}, d\right) \in \mathcal{D}$. So let $C>1 /(4 \varsigma)$ and let $\beta$ and $\tau$ be as in the first step. It then follows from (4.21) by the cocycle property and induction that

$$
\tilde{E}\left(\tilde{x}\left(n \tau, \tilde{x}_{0}, d\right)\right) \leq \beta^{n} \tilde{E}\left(\tilde{x}_{0}\right)+C\|d\|_{[0, n \tau], 2}^{2}
$$


for every $n \in \mathbb{N}_{0}$ and every $\left(\tilde{x}_{0}, d\right) \in \mathcal{D}$. If now $t \in[0, \infty)$ is arbitrary, then

$$
t=n \tau+t-n \tau \quad \text { and } \quad t-n \tau \in[0, \tau)
$$

for a unique $n=n_{t} \in \mathbb{N}_{0}$ and thus it follows by the cocycle property and by (4.32) and (3.17) that

$$
\begin{aligned}
\tilde{E}\left(\tilde{x}\left(t, \tilde{x}_{0}, d\right)\right) & \leq \beta^{n} \tilde{E}\left(\tilde{x}\left(t-n \tau, \tilde{x}_{0}, d\right)\right)+C\|d(\cdot+t-n \tau)\|_{[0, n \tau], 2}^{2} \\
& \leq \beta^{n} \tilde{E}\left(\tilde{x}_{0}\right)+\frac{1}{4 \varsigma}\|d\|_{[0, t-n \tau], 2}^{2}+C\|d\|_{[t-n \tau, t], 2}^{2}
\end{aligned}
$$

every $\left(\tilde{x}_{0}, d\right) \in \mathcal{D}$, from which (4.31) and hence the second step follows. We conclude by density and continuity that for every $C>1 /(4 \varsigma)$ there is a constant $\beta \in(0,1)$ and a time $\tau \in(0, \infty)$ such that (4.31) also holds for arbitrary data $\left(\tilde{x}_{0}, d\right) \in \tilde{X} \times L^{2}\left([0, \infty), \mathbb{R}^{k}\right)$ and $t \in[0, \infty)$. So, by $(2.18)$

$$
\left\|\tilde{x}\left(t, \tilde{x}_{0}, d\right)\right\| \leq \underline{\psi}^{-1}\left(\frac{2}{\beta} \beta^{t / \tau} \bar{\psi}\left(\left\|\tilde{x}_{0}\right\|\right)\right)+\underline{\psi}^{-1}\left(2 C\|d\|_{[0, t], 2}^{2}\right)
$$

for every $t \in[0, \infty)$ and every $\left(\tilde{x}_{0}, d\right) \in \tilde{X} \times L^{2}\left([0, \infty), \mathbb{R}^{k}\right)$. In particular, $\tilde{\mathfrak{S}}$ is of uniform asymptotic gain $\bar{\gamma}:=\underline{\psi}^{-1}\left(2 C \cdot{ }^{2}\right)$ for every $C>1 /(4 \varsigma)$, as desired.

\subsection{Weak input-to-state stability of the closed-loop system}

In this section we show that for systems $\mathfrak{S}$ of arbitrary order $N \in \mathbb{N}$ and for a general class of controllers $\mathfrak{S}_{c}$, the closed-loop system $\tilde{\mathfrak{S}}$ is weakly input-to-state stable. We call $\tilde{\mathfrak{S}}$ weakly input-to-state stable w.r.t. inputs from $L^{2}\left([0, \infty), \mathbb{R}^{k}\right)$ iff it is uniformly globally stable and of weak asymptotic gain. See [45] for other characterizations of weak input-to-state stability. In this context, the weak asymptotic gain property by definition means the following: there is a function $\bar{\gamma} \in \mathcal{K} \cup\{0\}$ such that for every $\varepsilon>0$ and every $\tilde{x}_{0} \in \tilde{X}$ and $d \in L^{2}\left([0, \infty), \mathbb{R}^{k}\right)$ there is a time $\bar{\tau}=\bar{\tau}\left(\varepsilon, \tilde{x}_{0}, d\right)$ such that

$$
\left\|\tilde{x}\left(t, \tilde{x}_{0}, d\right)\right\| \leq \varepsilon+\bar{\gamma}\left(\|d\|_{2}\right) \quad(t \geq \bar{\tau})
$$

A function $\bar{\gamma}$ as above is called a weak (asymptotic) gain (function) for $\tilde{\mathfrak{S}}$. We observe that the only difference to the uniform asymptotic gain property is that the time $\bar{\tau}$ is allowed to depend on the initial state $\tilde{x}_{0}$ (instead of only on its norm) and on the disturbance $d$. In [30] the weak asymptotic gain property is called just asymptotic gain property. Also, the weak asymptotic gain property with weak gain 0 is often just termed global attractivity [30, 31].

Lemma 4.8. If the assumptions (Conditions 2.1, 2.2, 3.1) of the solvability results are satisfied and $\tilde{\mathfrak{S}}$ is weakly input-to-state stable w.r.t. inputs from $L^{2}\left([0, \infty), \mathbb{R}^{k}\right)$, then for every $\left(\tilde{x}_{0}, d\right) \in \tilde{X} \times L^{2}\left([0, \infty), \mathbb{R}^{k}\right)$ one has

$$
\tilde{x}\left(t, \tilde{x}_{0}, d\right) \longrightarrow 0 \quad(t \rightarrow \infty) .
$$

Proof. Completely analogous to the proof of Lemma 4.2, the only difference being that there the convergence (4.3) was even locally uniform w.r.t. $\tilde{x}_{0}$.

In order to achieve weak input-to-state stability, we add the following conditions on the system $\mathfrak{S}$ and the controller $\mathfrak{S}_{c}$ to the assumptions from the solvability results.

Condition 4.9. $\mathfrak{S}$ is classically approximately observable in infinite time, that is, for non-zero initial state $0 \neq x_{0} \in D(\mathcal{A}) \cap \operatorname{ker} \mathcal{B}=D(A)$ and zero input $u=0$, the corresponding classical output $y\left(\cdot, x_{0}, 0\right):=\mathcal{C} x\left(\cdot, x_{0}, 0\right)$ of $\mathfrak{S}$ cannot vanish identically on $[0, \infty)$. 
Condition 4.10. (i) $\mathcal{R}$ is strictly damping, that is, for some constants $\underline{c}, \bar{c}, \delta>0$,

$$
v_{2}^{\top} \mathcal{R}\left(v_{2}\right) \geq \underline{c}\left|v_{2}\right|^{2} \quad\left(\left|v_{2}\right| \leq \delta\right) \quad \text { and } \quad v_{2}^{\top} \mathcal{R}\left(v_{2}\right) \geq \bar{c} \quad\left(\left|v_{2}\right|>\delta\right)
$$

(ii) $B_{c}$, the input operator of the controller, is injective.

Theorem 4.11. Suppose that the assumptions (Conditions 2.1, 2.2, 3.1) of the solvability theorems are satisfied along with Conditions 4.9 and 4.10. Suppose further that the only equilibrium point $\tilde{x}_{*}$ of the undisturbed system

$$
\tilde{x}^{\prime}=\tilde{\mathcal{A}} \tilde{x}+\tilde{f}(\tilde{x}) \quad \text { with } \quad 0=\tilde{\mathcal{B}} \tilde{x}(t)
$$

is the point $\tilde{x}_{*}=0$. Then the closed-loop system $\tilde{\mathfrak{S}}$ is weakly input-to-state stable and the function $\bar{\gamma}=0$ is a weak asymptotic gain for $\tilde{\mathfrak{S}}$. In particular,

$$
\tilde{x}\left(t, \tilde{x}_{0}, d\right) \longrightarrow 0 \quad(t \rightarrow \infty)
$$

for every $\tilde{x}_{0} \in \tilde{X}$ and $d \in L^{2}\left([0, \infty), \mathbb{R}^{k}\right)$.

We now turn to the proof of the theorem and, for that purpose, it will be most helpful to write the nonlinear part of (3.1) as

$$
\tilde{f}(\tilde{x})=\tilde{B} g\left(\tilde{B}^{*} \tilde{x}\right)+\tilde{B} h(\tilde{C} \tilde{x}),
$$

where $\tilde{B}: \mathbb{R}^{m_{c}} \rightarrow \tilde{X}, \tilde{C}: \tilde{X} \rightarrow \mathbb{R}^{m_{c}}$ and $g, h: \mathbb{R}^{m_{c}} \rightarrow \mathbb{R}^{m_{c}}$ are such that

$$
\begin{gathered}
\tilde{B} v_{2}:=\left(0,0, v_{2}\right), \quad \tilde{C} \tilde{x}:=v_{1}, \\
g\left(v_{2}\right):=-\mathcal{R}\left(v_{2}\right) \quad \text { and } \quad h\left(v_{1}\right):=v_{1}-\nabla \mathcal{P}\left(v_{1}\right) .
\end{gathered}
$$

In particular, $\tilde{B}^{*} \tilde{x}=K v_{2}$ for every $\tilde{x}=\left(x, v_{1}, v_{2}\right) \in \tilde{X}$. We begin by rewriting the closed-loop equation (3.1) as

$$
\begin{gathered}
\tilde{x}^{\prime}=\left(\tilde{\mathcal{A}}-\lambda \tilde{B} \tilde{B}^{*}\right) \tilde{x}+\tilde{B}\left(\lambda \tilde{B}^{*} \tilde{x}+g\left(\tilde{B}^{*} \tilde{x}\right)\right)+\tilde{B} h(\tilde{C} \tilde{x}) \quad \text { and } \quad \tilde{x}(0)=\tilde{x}_{0} \\
d(t)=\tilde{\mathcal{B}} \tilde{x}(t),
\end{gathered}
$$

that is, as a perturbation of the respective linear boundary control system

$$
\tilde{x}^{\prime}=\left(\tilde{\mathcal{A}}-\lambda \tilde{B} \tilde{B}^{*}\right) \tilde{x} \quad \text { with } \quad d(t)=\tilde{\mathcal{B}} \tilde{x}(t),
$$

where $\lambda>0$. It follows from (4.39) by the transformation (3.3) and variation of constants that classical solutions of (3.1) satisfy the following integral equation:

$$
\begin{aligned}
\tilde{x}\left(t, \tilde{x}_{0}, d\right)=\mathrm{e}^{\left(\tilde{A}-\lambda \tilde{B} \tilde{B}^{*}\right) t} \tilde{x}_{0} & +\int_{0}^{t} \mathrm{e}^{\left(\tilde{A}-\lambda \tilde{B} \tilde{B}^{*}\right)(t-s)} \tilde{B}\left(\lambda \tilde{B}^{*} \tilde{x}\left(s, \tilde{x}_{0}, d\right)+g\left(\tilde{B}^{*} \tilde{x}\left(s, \tilde{x}_{0}, d\right)\right)\right) \mathrm{d} s \\
& +\int_{0}^{t} \mathrm{e}^{\left(\tilde{A}-\lambda \tilde{B} \tilde{B}^{*}\right)(t-s)} \tilde{B} h\left(\tilde{C} \tilde{x}\left(s, \tilde{x}_{0}, d\right)\right) \mathrm{d} s+\Phi_{t}^{\lambda}(d)
\end{aligned}
$$

for every $\left(\tilde{x}_{0}, d\right) \in \mathcal{D}$, where

$$
\Phi_{t}^{\lambda}(d):=-\mathrm{e}^{\left(\tilde{A}-\lambda \tilde{B} \tilde{B}^{*}\right) t} \tilde{R} d(0)+\tilde{R} d(t)+\int_{0}^{t} \mathrm{e}^{\left(\tilde{A}-\lambda \tilde{B} \tilde{B}^{*}\right)(t-s)}\left(\left(\tilde{\mathcal{A}}-\lambda \tilde{B} \tilde{B}^{*}\right) \tilde{R} d(s)-\tilde{R} d^{\prime}(s)\right) \mathrm{d} s .
$$




\subsubsection{Central ingredients of the proof}

A first important ingredient to proceed from (4.41) is the following approximate observability result for the collocated linear system $\mathfrak{S}\left(\tilde{A}, \tilde{B}, \tilde{B}^{*}\right)$.

Lemma 4.12. Under the assumptions of the above theorem, the linear system $\mathfrak{S}\left(\tilde{A}, \tilde{B}, \tilde{B}^{*}\right)$ is approximately observable in infinite time.

Proof. Suppose that for some $\tilde{x}_{0} \in \tilde{X}$ we have

$$
\tilde{B}^{*} \mathrm{e}^{\tilde{A} t} \tilde{x}_{0}=0 \quad(t \in[0, \infty))
$$

We then have to show that $\tilde{x}_{0}=0$. Setting

$$
\left(x_{n 0}, v_{1 n 0}, v_{2 n 0}\right):=\tilde{x}_{n 0}:=n \int_{0}^{1 / n} \mathrm{e}^{\tilde{A} s} \tilde{x}_{0} \mathrm{~d} s
$$

for $n \in \mathbb{N}$, we have of course that

$$
\tilde{x}_{n 0} \in D(\tilde{A}) \quad(n \in \mathbb{N}) \quad \text { and } \quad \tilde{x}_{n 0} \longrightarrow \tilde{x}_{0} \quad(n \rightarrow \infty)
$$

and in view of (4.43) we also have that

$$
v_{2 n}(t)=\tilde{B}^{*} \mathrm{e}^{\tilde{A} t} \tilde{x}_{n 0}=0 \quad(n \in \mathbb{N}, t \in[0, \infty)),
$$

where we used the notation $\left(x_{n}(t), v_{1 n}(t), v_{2 n}(t)\right):=\tilde{x}_{n}(t):=\mathrm{e}^{\tilde{A} t} \tilde{x}_{n 0}$. We will also use the abbreviations

$$
u_{n}(t):=\mathcal{B} x_{n}(t) \quad \text { and } \quad y_{n}(t):=\mathcal{C} x_{n}(t)
$$

in the following. Choose and fix now $n \in \mathbb{N}$. We know from (4.44.a) that $\tilde{x}_{n}=\mathrm{e}^{\tilde{A} \cdot} \tilde{x}_{n 0}$ is continuously differentiable with

$$
\tilde{x}_{n}^{\prime}(t)=\left(\begin{array}{c}
x_{n}^{\prime}(t) \\
v_{1 n}^{\prime}(t) \\
v_{2 n}^{\prime}(t)
\end{array}\right)=\tilde{A}\left(\begin{array}{c}
x_{n}(t) \\
v_{1 n}(t) \\
v_{2 n}(t)
\end{array}\right)=\left(\begin{array}{c}
\mathcal{A} x_{n}(t) \\
K v_{2 n}(t) \\
-v_{1 n}(t)+B_{c} y_{n}(t)
\end{array}\right)
$$

for all $t \in[0, \infty)$. So, by $(4.45)$ it follows that $v_{1 n}=v_{1 n 0}$ is constant and that

$$
0=v_{2 n}^{\prime}(t)=-v_{1 n 0}+B_{c} y_{n}(t) \quad(t \in[0, \infty)) .
$$

And from this, in turn, it follows by the injectivity of $B_{c}$ (Condition 4.10 (ii)) that

$$
y_{n}(t)=L_{c} v_{1 n 0}=: y_{n 0} \quad(t \in[0, \infty)),
$$

where $L_{c}$ is an arbitrary left-inverse of $B_{c}$. We also know from (4.44.a) that $\tilde{x}_{n}(t)=\mathrm{e}^{\tilde{A} t} \tilde{x}_{n 0} \in D(\tilde{A}) \subset$ ker $\tilde{\mathcal{B}}$ for all $t$ and so by (4.45) and (4.48) it follows that

$$
0=\tilde{\mathcal{B}} \tilde{x}_{n}(t)=\mathcal{B} x_{n}(t)+B_{c}^{\top} K v_{2 n}(t)+S_{c} \mathcal{C} x_{n}(t)=u_{n}(t)+S_{c} y_{n 0} \quad(t \in[0, \infty)) .
$$


Since $\mathfrak{S}$ is impedance-passive (Condition 2.1) and since $x_{n}=x\left(\cdot, x_{n 0}, u_{n}\right.$ ) by the uniqueness statement made around (2.10), it further follows using (4.48) and (4.49) that

$$
0 \leq E\left(x_{n}(t)\right) \leq E\left(x_{n 0}\right)+\int_{0}^{t} u_{n}(s)^{\top} y_{n}(s) \mathrm{d} s=E\left(x_{n 0}\right)-\left(y_{n 0}^{\top} S_{c} y_{n 0}\right) t
$$

for all $t \in[0, \infty)$. So, by the positive definiteness of $S_{c}$, we see that $y_{n 0}=0$ and thus by (4.48), (4.47), (4.49) that

$$
y_{n}(t)=0 \quad \text { and } \quad v_{1 n 0}=0 \quad \text { and } \quad u_{n}(t)=0
$$

for all $t \in[0, \infty)$. Since now $\mathfrak{S}$ is classically approximately observable in infinite time (Condition 4.9) and since $y_{n}=y\left(\cdot, x_{n 0}, u_{n}\right)$, we conclude by (4.50.a) and (4.50.c) that

$$
x_{n 0}=0 .
$$

Combining (4.45), (4.50.b), (4.51) with (4.44.b), we finally get $\tilde{x}_{0}=0$ as desired.

A second important ingredient is the following stabilization result for the collocated linear system $\mathfrak{S}\left(\tilde{A}, \tilde{B}, \tilde{B}^{*}\right)$, which hinges on the approximate observability property just established (Lem. 4.12$)$ and on the compactness of the resolvent of $\tilde{A}$ (Lem. 2.3).

Lemma 4.13. Under the assumptions of the above theorem, one has for every $\lambda \in(0, \infty)$ :

(i) the linear operator

$$
L^{2}\left([0, \infty), \mathbb{R}^{m_{c}}\right) \ni u \mapsto \int_{0}^{\infty} \mathrm{e}^{\left(\tilde{A}-\lambda \tilde{B} \tilde{B}^{*}\right) s} \tilde{B} u(s) \mathrm{d} s \in \tilde{X}
$$

is well-defined and bounded with operator norm less than or equal to 1

(ii) the semigroup $\mathrm{e}^{\left(\tilde{A}-\lambda \tilde{B} \tilde{B}^{*}\right)}$ is strongly stable and $\left(\tilde{A}-\lambda \tilde{B} \tilde{B}^{*}\right)^{-1}$ is a compact operator in $\tilde{X}$

(iii) for every $u \in L^{2}\left([0, \infty), \mathbb{R}^{m_{c}}\right)$,

$$
\int_{0}^{t} \mathrm{e}^{\left(\tilde{A}-\lambda \tilde{B} \tilde{B}^{*}\right)(t-s)} \tilde{B} u(s) \mathrm{d} s \longrightarrow 0 \quad(t \rightarrow \infty)
$$

(iv) for every $u \in A C_{\mathrm{loc}}\left([0, \infty), \mathbb{R}^{m_{c}}\right) \cap L^{\infty}\left([0, \infty), \mathbb{R}^{m_{c}}\right)$ with $u^{\prime} \in L^{2}\left([0, \infty), \mathbb{R}^{m_{c}}\right)$ and every sequence $\left(t_{n}\right)$ with $t_{n} \longrightarrow \infty$, there is a subsequence $\left(t_{n_{l}}\right)$ such that the following limits exist and conincide:

$$
\lim _{l \rightarrow \infty} \int_{0}^{t_{n_{l}}} \mathrm{e}^{\left(\tilde{A}-\lambda \tilde{B} \tilde{B}^{*}\right)\left(t_{n_{l}}-s\right)} \tilde{B} u(s) \mathrm{d} s=-\lim _{l \rightarrow \infty}\left(\tilde{A}-\lambda \tilde{B} \tilde{B}^{*}\right)^{-1} \tilde{B} u\left(t_{n_{l}}\right)
$$

Proof. Since $\mathfrak{S}\left(\tilde{A}, \tilde{B}, \tilde{B}^{*}\right)$ is approximately observable in infinite time (Lem. 4.12), assertion (i), the strong stability part of assertion (ii), and assertion (iii) follow by a well-known stability result for collocated systems. See [5] or [38] (Lem. 2.2.6 and 2.1.3) or [48] (Sect. 3.1), for instance. Also, by the strong stability of $\mathrm{e}^{\left(\tilde{A}-\lambda \tilde{B} \tilde{B}^{*}\right)}$. and the compactness of the resolvent of $\tilde{A}$ and hence $\tilde{A}-\lambda \tilde{B} \tilde{B}^{*}$ (Lem. 2.3), we have

$$
0 \notin \sigma_{p}\left(\tilde{A}-\lambda \tilde{B} \tilde{B}^{*}\right)=\sigma\left(\tilde{A}-\lambda \tilde{B} \tilde{B}^{*}\right) .
$$


So, $\left(\tilde{A}-\lambda \tilde{B} \tilde{B}^{*}\right)^{-1}$ exists and is compact. And finally, assertion (iv) follows by partial integration:

$$
\begin{aligned}
\int_{0}^{t_{n}} \mathrm{e}^{\left(\tilde{A}-\lambda \tilde{B} \tilde{B}^{*}\right)\left(t_{n}-s\right)} \tilde{B} u(s) \mathrm{d} s= & -\left.\left(\tilde{A}-\lambda \tilde{B} \tilde{B}^{*}\right)^{-1} \mathrm{e}^{\left(\tilde{A}-\lambda \tilde{B} \tilde{B}^{*}\right)\left(t_{n}-s\right)} \tilde{B} u(s)\right|_{s=0} ^{s=t_{n}} \\
& +\left(\tilde{A}-\lambda \tilde{B} \tilde{B}^{*}\right)^{-1} \int_{0}^{t_{n}} \mathrm{e}^{\left(\tilde{A}-\lambda \tilde{B} \tilde{B}^{*}\right)\left(t_{n}-s\right)} \tilde{B} u^{\prime}(s) \mathrm{d} s
\end{aligned}
$$

and by then exploiting assertions (ii) and (iii).

Lemma 4.14. Under the assumptions of the above theorem, one has for every $\tilde{x}_{0} \in X$ and $d \in L^{2}\left([0, \infty), \mathbb{R}^{k}\right)$ :

(i) $\tilde{B}^{*} \tilde{x}\left(\cdot, \tilde{x}_{0}, d\right)$ and $g \circ\left(\tilde{B}^{*} \tilde{x}\left(\cdot, \tilde{x}_{0}, d\right)\right)$ belong to $L^{2}\left([0, \infty), \mathbb{R}^{m_{c}}\right)$.

(ii) $h \circ\left(\tilde{C} \tilde{x}\left(\cdot, \tilde{x}_{0}, d\right)\right)$ belongs to $A C_{\mathrm{loc}}\left([0, \infty), \mathbb{R}^{m_{c}}\right) \cap L^{\infty}\left([0, \infty), \mathbb{R}^{m_{c}}\right)$ and the derivative $\left(h \circ\left(\tilde{C} \tilde{x}\left(\cdot, \tilde{x}_{0}, d\right)\right)\right)^{\prime}$ belongs to $L^{2}\left([0, \infty), \mathbb{R}^{m_{c}}\right)$.

Proof. In the entire proof, we adopt the short-hand notation $\rho\left(\tilde{x}_{0}, d\right)$ for $\tilde{x}_{0} \in \tilde{X}$ and $d \in L^{2}\left([0, \infty), \mathbb{R}^{k}\right)$ from the proof of Lemma 3.3. We also denote by $L_{g}(\rho)$ and $L_{h}(\rho)$ for $\rho \in[0, \infty)$ Lipschitz constants of $\left.g\right|_{\bar{B}_{\rho}(0)}$ and $\left.h\right|_{\bar{B}_{\rho}(0)}$ such that $\rho \mapsto L_{g}(\rho), L_{h}(\rho)$ are continuous and monotonically increasing.

(i) We first show that for every $\left(\tilde{x}_{0}, d\right) \in \tilde{X} \times L^{2}\left([0, \infty), \mathbb{R}^{k}\right)$ and every $t \in[0, \infty)$ one has the following estimates:

$$
\begin{gathered}
\left\|\tilde{B}^{*} \tilde{x}\left(\cdot, \tilde{x}_{0}, d\right)\right\|_{[0, t], 2}^{2} \leq\left(1 / \underline{c}+\|K\| \rho\left(\tilde{x}_{0}, d\right)^{2} / \bar{c}\right)\left(\tilde{E}\left(\tilde{x}_{0}\right)+\frac{1}{4 \varsigma}\|d\|_{[0, t], 2}^{2}\right) \\
\left\|g \circ\left(\tilde{B}^{*} \tilde{x}\left(\cdot, \tilde{x}_{0}, d\right)\right)\right\|_{[0, t], 2}^{2} \leq L_{g}\left(\|K\|^{1 / 2} \rho\left(\tilde{x}_{0}, d\right)\right)^{2}\left(1 / \underline{c}+\|K\| \rho\left(\tilde{x}_{0}, d\right)^{2} / \bar{c}\right)\left(\tilde{E}\left(\tilde{x}_{0}\right)+\frac{1}{4 \varsigma}\|d\|_{[0, t], 2}^{2}\right) .
\end{gathered}
$$

Since both sides of (4.52) and (4.53) are continuous w.r.t. $\left(\tilde{x}_{0}, d\right) \in \tilde{X} \times L^{2}\left([0, \infty), \mathbb{R}^{k}\right)$ by Corollary 3.5 , it is sufficient to prove these estimates only for all classical data $\left(\tilde{x}_{0}, d\right) \in \mathcal{D}$. So let $\left(\tilde{x}_{0}, d\right) \in \mathcal{D}$ and $t \in[0, \infty)$ and write $\left(x, v_{1}, v_{2}\right):=\tilde{x}:=\tilde{x}\left(\cdot, \tilde{x}_{0}, d\right)$, so that $\tilde{B}^{*} \tilde{x}\left(\cdot, \tilde{x}_{0}, d\right)=K v_{2}$. It follows by Condition 4.10 (i) that

$$
\begin{aligned}
\left\|\tilde{B}^{*} \tilde{x}\left(\cdot, \tilde{x}_{0}, d\right)\right\|_{[0, t], 2}^{2} & =\int_{0}^{t}\left|K v_{2}(s)\right|^{2} \mathrm{~d} t=\int_{J_{\leq \delta}^{t}}\left|K v_{2}(s)\right|^{2} \mathrm{~d} t+\int_{J_{>\delta}^{t}}\left|K v_{2}(s)\right|^{2} \mathrm{~d} t \\
& \leq\left(1 / \underline{c}+\|K\| \rho\left(\tilde{x}_{0}, d\right)^{2} / \bar{c}\right) \int_{0}^{t}\left(K v_{2}(s)\right)^{\top} \mathcal{R}\left(K v_{2}(s)\right) \mathrm{d} s
\end{aligned}
$$

for every $t \in[0, \infty)$, where we used the abbreviations

$$
J_{\leq \delta}^{t}:=\left\{s \in[0, t]:\left|K v_{2}(s)\right| \leq \delta\right\} \quad \text { and } \quad J_{>\delta}^{t}:=\left\{s \in[0, t]:\left|K v_{2}(s)\right|>\delta\right\}
$$

as well as the fact that

$$
\sup _{s \in[0, \infty)}\left|K v_{2}(s)\right|^{2} \leq\|K\| \sup _{s \in[0, \infty)}\left\|\tilde{x}\left(s, \tilde{x}_{0}, d\right)\right\|^{2} \leq\|K\| \rho\left(\tilde{x}_{0}, d\right)^{2}
$$

(Thm. 3.2 (ii)). It further follows from (3.16) with $\alpha:=1 /(2 \varsigma)$ that

$$
\int_{0}^{t}\left(K v_{2}(s)\right)^{\top} \mathcal{R}\left(K v_{2}(s)\right) \mathrm{d} s \leq \tilde{E}\left(\tilde{x}_{0}\right)+\frac{1}{4 \varsigma}\|d\|_{[0, t], 2}^{2}
$$


for every $t \in[0, \infty)$. Combining (4.54) and (4.56) we obtain (4.52). And since $g(0)=0$ we we also obtain (4.53) using (4.55). Assertion (i) now follows by letting $t \rightarrow \infty$ in (4.52) and (4.53).

(ii) Choose and fix $\tilde{x}_{0} \in \tilde{X}$ and $d \in L^{2}\left([0, \infty), \mathbb{R}^{k}\right)$ and write $\left(x, v_{1}, v_{2}\right):=\tilde{x}:=\tilde{x}\left(\cdot, \tilde{x}_{0}, d\right)$, so that $\tilde{C} \tilde{x}\left(\cdot, \tilde{x}_{0}, d\right)=v_{1}$. Since $\tilde{x}\left(\cdot, \tilde{x}_{0}, d\right)$ is the locally uniform limit of classical solutions by definition (3.38), we see that

$$
v_{1}(t)=v_{1}(0)+\int_{0}^{t} K v_{2}(s) \mathrm{d} s
$$

for all $t \in[0, \infty)$ and hence $v_{1}$ is locally Lipschitz continuous (even continuously differentiable). So, since $h$ is locally Lipschitz continuous as well and since

$$
\sup _{s \in[0, \infty)}\left|v_{1}(s)\right| \leq \sup _{s \in[0, \infty)}\left\|\tilde{x}\left(s, \tilde{x}_{0}, d\right)\right\| \leq \rho\left(\tilde{x}_{0}, d\right)
$$

by Theorem 4.1, it follows that the composition $h \circ v_{1}$ is locally Lipschitz continuous and bounded. In particular, $h \circ\left(\tilde{C} \tilde{x}\left(\cdot, \tilde{x}_{0}, d\right)\right)=h \circ v_{1}$ belongs to $A C_{\text {loc }}\left([0, \infty), \mathbb{R}^{m_{c}}\right) \cap L^{\infty}\left([0, \infty), \mathbb{R}^{m_{c}}\right)$ and thus is differentiable almost everywhere. In view of (4.58) and (4.57) its derivative satisfies

$$
\begin{aligned}
\left|\left(h \circ v_{1}\right)^{\prime}(s)\right| & =\lim _{\tau \rightarrow 0}\left|\frac{h\left(v_{1}(s+\tau)\right)-h\left(v_{1}(s)\right)}{\tau}\right| \leq L_{h}\left(\rho\left(\tilde{x}_{0}, d\right)\right) \lim _{\tau \rightarrow 0}\left|\frac{v_{1}(s+\tau)-v_{1}(s)}{\tau}\right| \\
& =L_{h}\left(\rho\left(\tilde{x}_{0}, d\right)\right)\left|\tilde{B}^{*} \tilde{x}\left(s, \tilde{x}_{0}, d\right)\right|
\end{aligned}
$$

for almost every $s \in[0, \infty)$. So,

$$
\left\|\left(h \circ\left(\tilde{C} \tilde{x}\left(\cdot, \tilde{x}_{0}, d\right)\right)\right)^{\prime}\right\|_{[0, t], 2}=\left\|\left(h \circ v_{1}\right)^{\prime}\right\|_{[0, t], 2} \leq L_{h}\left(\rho\left(\tilde{x}_{0}, d\right)\right)\left\|\tilde{B}^{*} \tilde{x}\left(\cdot, \tilde{x}_{0}, d\right)\right\|_{[0, t], 2}
$$

for every $t \in[0, \infty)$. In particular, $\left(h \circ\left(\tilde{C} \tilde{x}\left(\cdot, \tilde{x}_{0}, d\right)\right)\right)^{\prime}=\left(h \circ v_{1}\right)^{\prime}$ belongs to $L^{2}\left([0, \infty), \mathbb{R}^{m_{c}}\right)$ by assertion (i), which concludes the proof of assertion (ii).

A third important ingredient is the following lemma which says that the linear boundary control system

$$
\tilde{x}^{\prime}=\left(\tilde{\mathcal{A}}-\lambda \tilde{B} \tilde{B}^{*}\right) \tilde{x} \quad \text { with } \quad d(t)=\tilde{\mathcal{B}} \tilde{x}(t)
$$

for $\lambda>0$ is infinite-time admissible w.r.t. inputs $d \in L^{2}\left([0, \infty), \mathbb{R}^{k}\right)$.

Lemma 4.15. Under the assumptions of the above theorem, the linear operator $\Phi_{t}^{\lambda}: C_{c}^{2}\left([0, \infty), \mathbb{R}^{k}\right) \rightarrow \tilde{X}$ defined by (4.42) can be (uniquely) extended to a bounded linear operator $\bar{\Phi}_{t}^{\lambda}: L^{2}\left([0, \infty), \mathbb{R}^{k}\right) \rightarrow \tilde{X}$ for every $t \in[0, \infty)$ and $\lambda \in(0, \infty)$ and

$$
\sup _{t \in[0, \infty)}\left\|\bar{\Phi}_{t}^{\lambda}\right\|<\infty
$$

Proof. Choose and fix $\lambda \in(0, \infty)$. We adopt the short-hand notations $\rho\left(\tilde{x}_{0}, d\right)$ and $L_{g}(\rho), L_{h}(\rho)$ from the proof of Lemma 4.14.

As a first step, we show that the restriction $\Phi_{t, 0}^{\lambda}$ of $\Phi_{t}^{\lambda}$ to

$$
C_{c, 0}^{2}\left([0, \infty), \mathbb{R}^{k}\right):=\left\{d \in C_{c}^{2}\left([0, \infty), \mathbb{R}^{k}\right): d(0)=0\right\}
$$


can be (uniquely) extended to a bounded linear operator $\bar{\Phi}_{t, 0}^{\lambda}: L^{2}\left([0, \infty), \mathbb{R}^{k}\right) \rightarrow \tilde{X}$ for every $t \in[0, \infty)$ and

$$
\sup _{t \in[0, \infty)}\left\|\bar{\Phi}_{t, 0}^{\lambda}\right\|<\infty
$$

So let $t \in[0, \infty)$. We see by $(4.41)$ that

$$
\begin{aligned}
\Phi_{t, 0}^{\lambda}(d)=\tilde{x}(t, 0, d) & -\int_{0}^{t} \mathrm{e}^{\left(\tilde{A}-\lambda \tilde{B} \tilde{B}^{*}\right)(t-s)} \tilde{B}\left(\lambda \tilde{B}^{*} \tilde{x}(s, 0, d)+g\left(\tilde{B}^{*} \tilde{x}(s, 0, d)\right)\right) \mathrm{d} s \\
& -\int_{0}^{t} \mathrm{e}^{\left(\tilde{A}-\lambda \tilde{B} \tilde{B}^{*}\right)(t-s)} \tilde{B} h(\tilde{C} \tilde{x}(s, 0, d)) \mathrm{d} s
\end{aligned}
$$

for every $d \in C_{c, 0}^{2}\left([0, \infty), \mathbb{R}^{k}\right)$. It follows by Theorem 3.2 (ii) that

$$
\|\tilde{x}(t, 0, d)\| \leq \underline{\gamma}\left(\|d\|_{[0, t], 2}\right)
$$

for all $d \in C_{c, 0}^{2}\left([0, \infty), \mathbb{R}^{k}\right)$. Also, it follows by Lemma 4.13 (i) together with (4.52), (4.53) that

$$
\begin{aligned}
& \left\|\int_{0}^{t} \mathrm{e}^{\left(\tilde{A}-\lambda \tilde{B} \tilde{B}^{*}\right)(t-s)} \tilde{B}\left(\lambda \tilde{B}^{*} \tilde{x}(s, 0, d)+g\left(\tilde{B}^{*} \tilde{x}(s, 0, d)\right)\right) \mathrm{d} s\right\| \\
& \quad \leq \lambda\left\|\tilde{B}^{*} \tilde{x}(\cdot, 0, d)\right\|_{[0, t], 2}+\left\|g \circ\left(\tilde{B}^{*} \tilde{x}(\cdot, 0, d)\right)\right\|_{[0, t], 2} \\
& \quad \leq\left(\lambda+L_{g}\left(\|K\|^{1 / 2} \rho(0, d)\right)\right)\left(1 / \underline{c}+\|K\| \rho(0, d)^{2} / \bar{c}\right)^{1 / 2}\left(\frac{1}{4 \varsigma}\right)^{1 / 2}\|d\|_{[0, t], 2}
\end{aligned}
$$

for all $d \in C_{c, 0}^{2}\left([0, \infty), \mathbb{R}^{k}\right)$. And finally, it follows by integration by parts (which is allowed by Lem. 4.14 (ii)) and by Lemma 4.13 (i) together with (4.59), (4.52) that

$$
\begin{aligned}
& \left\|\int_{0}^{t} \mathrm{e}^{\left(\tilde{A}-\lambda \tilde{B} \tilde{B}^{*}\right)(t-s)} \tilde{B} h(\tilde{C} \tilde{x}(s, 0, d)) \mathrm{d} s\right\| \leq \\
& \quad+\left\|\left(\tilde{A}-\lambda \tilde{B} \tilde{B}^{*}\right)^{-1} \tilde{B} h(\tilde{C} \tilde{x}(t, 0, d))\right\| \\
& +\left(\tilde{A}-\lambda \tilde{B} \tilde{B}^{*}\right)^{-1} \int_{0}^{t} \mathrm{e}^{\left(\tilde{A}-\lambda \tilde{B} \tilde{B}^{*}\right)(t-s)} \tilde{B}(h \circ(\tilde{C} \tilde{x}(\cdot, 0, d)))^{\prime}(s) \mathrm{d} s \| \\
& \leq\left\|\left(\tilde{A}-\lambda \tilde{B} \tilde{B}^{*}\right)^{-1}\right\|\left(\|\tilde{B}\| L_{h}(\rho(0, d))\|\tilde{x}(t, 0, d)\|+\left\|(h \circ(\tilde{C} \tilde{x}(\cdot, 0, d)))^{\prime}\right\|_{[0, t], 2}\right) \\
& \leq\left\|\left(\tilde{A}-\lambda \tilde{B} \tilde{B}^{*}\right)^{-1}\right\| L_{h}(\rho(0, d))\left(\|\tilde{B}\| \underline{\gamma}\left(\|d\|_{[0, t], 2}\right)+\left(1 / \underline{c}+\|K\| \rho(0, d)^{2} / \bar{c}\right)^{1 / 2}\left(\frac{1}{4 \varsigma}\right)^{1 / 2}\|d\|_{[0, t], 2}\right)
\end{aligned}
$$

for all $d \in C_{c, 0}^{2}\left([0, \infty), \mathbb{R}^{k}\right)$. Combining now (4.63) and (4.64), (4.65), (4.66) we see that

$$
\left\|\bar{\Phi}_{t, 0}^{\lambda}(d)\right\| \leq C_{\lambda}(\rho(0, d))\left(\|d\|_{[0, t], 2}+\underline{\gamma}\left(\|d\|_{[0, t], 2}\right)\right)
$$

for all $d \in C_{c, 0}^{2}\left([0, \infty), \mathbb{R}^{k}\right)$, where $[0, \infty) \ni \rho \mapsto C_{\lambda}(\rho)$ is a continuous monotonically increasing function. It follows that

$$
\Phi_{t, 0}^{\lambda}: C_{c, 0}^{2}\left([0, \infty), \mathbb{R}^{k}\right) \rightarrow \tilde{X}
$$


is a linear operator that is bounded w.r.t. the norm of $L^{2}\left([0, \infty), \mathbb{R}^{k}\right)$ and thus, by the density of $C_{c, 0}^{2}\left([0, \infty), \mathbb{R}^{k}\right)$ in $L^{2}\left([0, \infty), \mathbb{R}^{k}\right)$, can be uniquely extended to a bounded linear operator $\bar{\Phi}_{t, 0}^{\lambda}: L^{2}\left([0, \infty), \mathbb{R}^{k}\right) \rightarrow \tilde{X}$. It further follows that

$$
\begin{aligned}
\sup _{t \in[0, \infty)}\left\|\bar{\Phi}_{t, 0}^{\lambda}\right\| & =\sup _{t \in[0, \infty)} \sup \left\{\left\|\bar{\Phi}_{t, 0}^{\lambda}(d)\right\|: d \in C_{c, 0}^{2}\left([0, \infty), \mathbb{R}^{k}\right) \text { with }\|d\|_{2} \leq 1\right\} \\
& \leq C_{\lambda}(\rho(0,1))(1+\underline{\gamma}(1))<\infty .
\end{aligned}
$$

As a second step, we observe that also the non-restricted operator $\Phi_{t}^{\lambda}$ can be (uniquely) extended to a bounded linear operator $\bar{\Phi}_{t}^{\lambda}: L^{2}\left([0, \infty), \mathbb{R}^{k}\right) \rightarrow \tilde{X}$ and that $\bar{\Phi}_{t}^{\lambda}=\bar{\Phi}_{t, 0}^{\lambda}$ for every $t \in[0, \infty)$. In order to do so, we have only to show that

$$
\bar{\Phi}_{t, 0}^{\lambda}(d)=\Phi_{t}^{\lambda}(d)
$$

for every $d \in C_{c}^{2}\left([0, \infty), \mathbb{R}^{k}\right)$. And this, in turn, can be achieved in the same way as in the second step of the proof of Lemma 3.3. (Instead of (3.28) the essential ingredient now is the estimate (4.67), for it again allows us to conclude $\Phi_{t / n, 0}^{\lambda}\left(d_{n}\right) \longrightarrow 0$ as $n \rightarrow \infty$ for every sequence $\left(d_{n}\right)$ as in (3.31).)

In conjunction with (4.62) the second step proves the lemma. We finally remark that the mere bounded extendability of $\Phi_{t}^{\lambda}$ (without the finiteness condition (4.61)) could alternatively also be concluded from Lemma 3.3 by a perturbation argument (for which the approximate observability of $\mathfrak{S}\left(\tilde{A}, \tilde{B}^{*}, \tilde{B}\right)$ would not be needed). See [48] for a collection of positive and negative perturbation results for (infinite-time) admissibility.

\subsubsection{Conclusion of the proof}

With the above lemmas at hand, we can now conclude the proof of weak input-to-state stability with weak asymptotic gain $\bar{\gamma}=0$ in three simple steps. Since we already know that the closed-loop system is uniformly globally stable (Thm. 4.1), we have only to show that

$$
\tilde{x}\left(t, \tilde{x}_{0}, d\right) \longrightarrow 0 \quad(t \rightarrow \infty)
$$

for every $\tilde{x}_{0} \in \tilde{X}$ and $d \in L^{2}\left([0, \infty), \mathbb{R}^{k}\right)$. So let $\left(\tilde{x}_{0}, d\right) \in \tilde{X} \times L^{2}\left([0, \infty), \mathbb{R}^{k}\right)$. It then follows from (4.41) by a simple approximation argument (using (3.38) and Lemma 4.15) that

$$
\begin{aligned}
\tilde{x}\left(t, \tilde{x}_{0}, d\right)=\mathrm{e}^{\left(\tilde{A}-\lambda \tilde{B} \tilde{B}^{*}\right) t} \tilde{x}_{0} & +\int_{0}^{t} \mathrm{e}^{\left(\tilde{A}-\lambda \tilde{B} \tilde{B}^{*}\right)(t-s)} \tilde{B}\left(\lambda \tilde{B}^{*} \tilde{x}\left(s, \tilde{x}_{0}, d\right)+g\left(\tilde{B}^{*} \tilde{x}\left(s, \tilde{x}_{0}, d\right)\right)\right) \mathrm{d} s \\
& +\int_{0}^{t} \mathrm{e}^{\left(\tilde{A}-\lambda \tilde{B} \tilde{B}^{*}\right)(t-s)} \tilde{B} h\left(\tilde{C} \tilde{x}\left(s, \tilde{x}_{0}, d\right)\right) \mathrm{d} s+\bar{\Phi}_{t}^{\lambda}(d)
\end{aligned}
$$

for all $t \in[0, \infty)$ and $\lambda \in(0, \infty)$. We now show in three steps that all four terms on the right-hand side of $(4.70)$ converge to 0 as $t \rightarrow \infty$.

As a first step, we observe from Lemma 4.13 (ii) and from Lemma 4.13 (iii) in conjunction with Lemma 4.14 (i) that the first term and the second term (the first integral) on the right-hand side of (4.70) converge to 0 as $t \rightarrow \infty$ for every $\lambda \in(0, \infty)$.

As a second step, we observe from Lemma 4.15 that the last term on the right-hand side of (4.70) converges to 0 as $t \rightarrow \infty$ for every $\lambda \in(0, \infty)$. Indeed, for $d_{0} \in C_{c}^{2}\left([0, \infty), \mathbb{R}^{k}\right)$ the convergence $\Phi_{t}^{\lambda}\left(d_{0}\right) \longrightarrow 0$ as $t \rightarrow \infty$ immediately follows from (4.42) by virtue of Lemma 4.13 (ii). And from this, in turn, the desired convergence $\bar{\Phi}_{t}^{\lambda}(d) \longrightarrow 0$ follows by density and Lemma 4.15 . 
As a third and last step, we show that also the third term (the second integral) on the right-hand side of (4.70) converges to 0 as $t \rightarrow \infty$ for every $\lambda \in(0, \infty)$. Choose an arbitrary sequence $\left(t_{n}\right)$ with $t_{n} \longrightarrow \infty$ as $n \rightarrow \infty$. Then by Lemma 4.13 (iv) in conjunction with Lemma 4.14 (ii) there exists a subsequence $\left(t_{n_{l}}\right)$ such that for both $\lambda=1$ and $\lambda=2$ one has:

$$
\lim _{l \rightarrow \infty} \int_{0}^{t_{n_{l}}} \mathrm{e}^{\left(\tilde{A}-\lambda \tilde{B} \tilde{B}^{*}\right)\left(t_{n_{l}}-s\right)} \tilde{B} h\left(\tilde{C} \tilde{x}\left(s, \tilde{x}_{0}, d\right)\right) \mathrm{d} s=-\lim _{l \rightarrow \infty}\left(\tilde{A}-\lambda \tilde{B} \tilde{B}^{*}\right)^{-1} \tilde{B} h\left(\tilde{C} \tilde{x}\left(t_{n_{l}}, \tilde{x}_{0}, d\right)\right) .
$$

Combining now (4.70) with the first and second step and with (4.71), we see that $\tilde{x}_{*}=\lim _{l \rightarrow \infty} \tilde{x}\left(t_{n_{l}}, \tilde{x}_{0}, d\right)$ exists in $\tilde{X}$ and that

$$
\tilde{x}_{*}=-\left(\tilde{A}-\lambda \tilde{B} \tilde{B}^{*}\right)^{-1} \tilde{B} h\left(\tilde{C} \tilde{x}_{*}\right)
$$

for both $\lambda=1$ and $\lambda=2$. So, $\tilde{x}_{*} \in D(\tilde{A})$ and

$$
\left(\tilde{A}-\tilde{B} \tilde{B}^{*}\right) \tilde{x}_{*}=-\tilde{B} h\left(\tilde{C} \tilde{x}_{*}\right)=\left(\tilde{A}-2 \tilde{B} \tilde{B}^{*}\right) \tilde{x}_{*} .
$$

It follows from this that on the one hand

$$
\tilde{B} \tilde{B}^{*} \tilde{x}_{*}=0 \quad \text { hence } \quad \tilde{B}^{*} \tilde{x}_{*}=0
$$

and on the other hand

$$
\tilde{A} \tilde{x}_{*}+\tilde{f}\left(\tilde{x}_{*}\right)=\tilde{A} \tilde{x}_{*}+\tilde{B} g\left(\tilde{B}^{*} \tilde{x}_{*}\right)+\tilde{B} h\left(\tilde{C} \tilde{x}_{*}\right)=\tilde{A} \tilde{x}_{*}+\tilde{B} h\left(\tilde{C} \tilde{x}_{*}\right)=0
$$

In other words, (4.74) and (4.75) say that $\tilde{x}_{*}$ is an equilibrium point of (4.37) and thus $\tilde{x}_{*}=0$ by assumption. So, summarizing we have shown that for every sequence $\left(t_{n}\right)$ with $t_{n} \longrightarrow \infty$ there exists a subsequence $\left(t_{n_{l}}\right)$ such that

$$
\lim _{l \rightarrow \infty} \tilde{x}\left(t_{n_{l}}, \tilde{x}_{0}, d\right)=\tilde{x}_{*}=0
$$

And from this, in turn, the asserted convergence (4.69) follows (and therefore, in view of the first two steps, also the third step follows).

\subsection{Some remarks on the assumptions}

In this section, we discuss specializations and generalizations of the assumptions from the weak input-to-state stability result (Thm. 4.11). In particular, we clarify their relation to the assumptions from the uniform inputto-state stability result (Thm. 4.5). As the following two lemmas show, the assumption on $\mathfrak{S}$ from Theorem 4.5 (Condition 4.3) is more or less - apart from some minor extra conditions which are often satisfied in applications a sufficient condition both for the approximate observability assumption (Condition 4.9) and for the equilibrium point assumption from Theorem 4.11. See the conference paper [43] for versions of the stability theorems above that are simplified accordingly. In our applied examples (Sect. 5), we will make ample use of this.

Lemma 4.16. Suppose that the assumptions (Conditions 2.1, 2.2, 3.1) of the solvability theorems are satisfied and, in addition, that $\mathfrak{S}$ is even impedance-energy-preserving meaning that (2.8) holds with equality. If then Condition 4.3 is satisfied, then so is Condition 4.9.

Proof. Suppose that Condition 4.3 is satisfied and that $x_{0} \in D(A)$ is such that

$$
y\left(t, x_{0}, 0\right)=\mathcal{C} x\left(t, x_{0}, 0\right)=0 \quad(t \in[0, \infty)) .
$$


Since $x\left(\cdot, x_{0}, 0\right)=\mathrm{e}^{A \cdot} x_{0}$ is a classical solution of $x^{\prime}=\mathcal{A} x$ with $t \mapsto\left\|x\left(t, x_{0}, 0\right)\right\|_{X}$ being monotonically decreasing and since Condition 4.3 is satisfied, there exist positive constants $C_{0}, t_{0}$ such that

$$
\begin{aligned}
\left\|x\left(t, x_{0}, 0\right)\right\|_{X}^{2} & \leq C_{0} \int_{0}^{t}\left|\left(\mathcal{H} x\left(s, x_{0}, 0\right)\right)(\eta)\right|^{2} \mathrm{~d} s \\
& \leq C_{0} / \kappa \int_{0}^{t}\left|\left(\mathcal{B} x\left(s, x_{0}, 0\right)\right)(\eta)\right|^{2}+\left|\left(\mathcal{C} x\left(s, x_{0}, 0\right)\right)(\eta)\right|^{2} \mathrm{~d} s
\end{aligned}
$$

for all $t \in\left[t_{0}, \infty\right)$ (Lem. 9.1.2 of [12] or, more precisely, the third step in the proof of Thm. 3.5 of [44]). So, as $\mathcal{B} x\left(\cdot, x_{0}, 0\right) \equiv 0$ and $\mathcal{C} x\left(\cdot, x_{0}, 0\right) \equiv 0$ by $(4.77)$, we conclude that

$$
x\left(t, x_{0}, 0\right)=0
$$

at least for all $t \in\left[t_{0}, \infty\right)$. Since by the assumed impedance-energy-preservation of $\mathfrak{S}$ and by (4.77) the energy $t \mapsto 1 / 2\left\|x\left(t, x_{0}, 0\right)\right\|_{X}^{2}$ is constant on the whole of $[0, \infty)$, it follows from (4.79) that $x_{0}=0$ as desired.

Lemma 4.17. Suppose that the assumptions (Conditions 2.1, 2.2, 3.1) of the solvability theorems are satisfied and, in addition, that 0 is the only critical point of the potential energy $\mathcal{P}$ of $\mathfrak{S}_{c}$. If then Condition 4.3 is satisfied, then the only equilibrium point $\tilde{x}_{*}$ of (4.37) is 0 .

Proof. Suppose that Condition 4.3 is satisfied and that $\tilde{x}_{*}=\left(x_{*}, v_{* 1}, v_{* 2}\right)$ is an equilibrium point of (4.37). Then $\tilde{x}_{*} \in D(\tilde{\mathcal{A}})$ with

$$
0=\tilde{\mathcal{A}} \tilde{x}_{*}+\tilde{f}\left(\tilde{x}_{*}\right)=\left(\begin{array}{c}
\mathcal{A} x_{*} \\
K v_{* 2} \\
B_{c} \mathcal{C} x_{*}-\nabla \mathcal{P}\left(v_{* 1}\right)-\mathcal{R}\left(K v_{* 2}\right)
\end{array}\right)=\left(\begin{array}{c}
\mathcal{A} x_{*} \\
K v_{* 2} \\
B_{c} \mathcal{C} x_{*}-\nabla \mathcal{P}\left(v_{* 1}\right)
\end{array}\right)
$$

and

$$
0=\tilde{\mathcal{B}} \tilde{x}_{*}=\mathcal{B} x_{*}+B_{c}^{\top} K v_{* 2}+S_{c} \mathcal{C} x_{*}=\mathcal{B} x_{*}+S_{c} \mathcal{C} x_{*} .
$$

It follows from (4.80) and (4.81) using the impedance-passivity of $\mathfrak{S}$ (Condition 2.1) that

$$
0=\left\langle x_{*}, \mathcal{A} x_{*}\right\rangle_{X} \leq\left(\mathcal{B} x_{*}\right)^{\top} \mathcal{C} x_{*}=-\left(\mathcal{C} x_{*}\right)^{\top} S_{c} \mathcal{C} x_{*} \leq 0
$$

So, by (2.15) and (4.81) we see that

$$
\mathcal{C} x_{*}=0 \quad \text { and } \quad \mathcal{B} x_{*}=0 .
$$

In view of (4.80) and our extra assumption on the critical points of $\mathcal{P}$, this implies that $v_{* 1}=0$ and it remains to show that $x_{*}=0$ as well. Since by (4.80) the constant function $t \mapsto x(t):=x_{*}$ is a classical solution of $x^{\prime}=\mathcal{A} x$ and since Condition 4.3 is satisfied, there exist positive constants $C_{0}, t_{0}$ such that

$$
\left\|x_{*}\right\|_{X}^{2}=\|x(t)\|_{X}^{2} \leq C_{0} \int_{0}^{t}|(\mathcal{H} x(s))(\eta)|^{2} \mathrm{~d} s \leq C_{0} / \kappa \int_{0}^{t}\left|\mathcal{B} x_{*}\right|^{2}+\left|\mathcal{C} x_{*}\right|^{2} \mathrm{~d} s
$$

for all $t \in\left[t_{0}, \infty\right)$ (Lem. 9.1.2 of [12] or, more precisely, the third step in the proof of Thm. 3.5 of [44]). In view of (4.83), this implies that $x_{*}=0$ as desired. 
An inspection of the proof of Theorem 4.11 reveals that Condition 4.9 and Condition 4.10 (ii) are needed only to obtain the approximate observability of $\mathfrak{S}\left(\tilde{A}, \tilde{B}, \tilde{B}^{*}\right)$ (Lem. 4.12) - for all the other lemmas and arguments, the following Condition 4.18 and Condition 4.10 (i) (along with Conditions 2.1, 2.2, 3.1, of course) are sufficient.

Condition 4.18. $\mathfrak{S}\left(\tilde{A}, \tilde{B}, \tilde{B}^{*}\right)$ is approximately observable in infinite time or approximately controllable in infinite time.

Consequently, the assertions of Theorem 4.11 remain true if we replace Condition 4.9 by the more general Condition 4.18 and omit Condition 4.10 (ii) (while leaving all other assumptions of the theorem unchanged). Yet, the thus modified assumptions are not much more general. In fact, by the following lemma, the modified assumptions entail all the original assumptions except, possibly, the not very restrictive injectivity assumption on $B_{c}$ (Condition 4.10 (ii)).

Lemma 4.19. Suppose that the assumptions (Conditions 2.1, 2.2, 3.1) of the solvability theorems are satisfied and that $\mathfrak{S}\left(\tilde{A}, \tilde{B}, \tilde{B}^{*}\right)$ is approximately observable in infinite time. Then Condition 4.9 is satisfied as well.

Proof. Suppose $x_{0} \in D(A)$ is such that the output $y\left(t, x_{0}, 0\right)=\mathcal{C} x\left(t, x_{0}, 0\right)=0$ for all $t \in[0, \infty)$. We have to show that $x_{0}=0$. Set $\tilde{x}_{0}:=\left(x_{0}, 0,0\right) \in \tilde{X}$ and $\tilde{x}(t):=\left(x\left(t, x_{0}, 0\right), 0,0\right)=\left(\mathrm{e}^{A t} x_{0}, 0,0\right) \in \tilde{X}$, then $\tilde{x}$ is continuously differentiable and, by the zero-output assumption,

$$
\tilde{x}(t) \in D(\tilde{\mathcal{A}}) \cap \operatorname{ker} \tilde{\mathcal{B}}=D(\tilde{A}) \quad \text { and } \quad \tilde{x}^{\prime}(t)=\tilde{A} \tilde{x}(t)
$$

for all $t \in[0, \infty)$. Consequently, $\tilde{x}(t)=\mathrm{e}^{\tilde{A} t} \tilde{x}_{0}$ and thus

$$
\tilde{B}^{*} \mathrm{e}^{\tilde{A} t} \tilde{x}_{0}=\tilde{B}^{*} \tilde{x}(t)=0 \quad(t \in[0, \infty)) .
$$

Since $\mathfrak{S}\left(\tilde{A}, \tilde{B}, \tilde{B}^{*}\right)$ is approximately observable in infinite time, we obtain $\tilde{x}_{0}=0$ as desired.

\section{Some APPlications}

In this section, we apply the uniform and the weak input-to-state stability results to a vibrating string and a Timoshenko beam.

Example 5.1. Consider a vibrating string $[2,12,53]$, that is, the transverse displacement $w(t, \zeta)$ of the string at position $\zeta \in(a, b)$ evolves according to the partial differential equation

$$
\rho(\zeta) \partial_{t}^{2} w(t, \zeta)=\partial_{\zeta}\left(T(\zeta) \partial_{\zeta} w(t, \zeta)\right)
$$

for $t \in[0, \infty), \zeta \in(a, b)$ (vibrating string equation) and the energy $E_{w}(t)$ of the string at time $t$ is given by

$$
E_{w}(t)=\frac{1}{2} \int_{a}^{b} \rho(\zeta)\left(\partial_{t} w(t, \zeta)\right)^{2}+T(\zeta)\left(\partial_{\zeta} w(t, \zeta)\right)^{2} \mathrm{~d} \zeta
$$

In these equations, $\rho, T$ are the mass density and the Young modulus of the string and they are assumed to be absolutely continuous and to be bounded below and above by positive finite constants. Also, assume that the string is clamped at its left end, that is,

$$
\partial_{t} w(t, a)=0 \quad(t \in[0, \infty))
$$


and that the control input $u(t)$ and observation output $y(t)$ are given respectively by the force and by the velocity at the right end of the string, that is,

$$
u(t)=T(b) \partial_{\zeta} w(t, b) \quad \text { and } \quad y(t)=\partial_{t} w(t, b)
$$

for all $t \in[0, \infty)$. With the choices

$$
x(t)(\zeta):=\left(\begin{array}{c}
\rho(\zeta) \partial_{t} w(t, \zeta) \\
\partial_{\zeta} w(t, \zeta)
\end{array}\right), \quad \mathcal{H}(\zeta):=\left(\begin{array}{cc}
1 / \rho(\zeta) & 0 \\
0 & T(\zeta)
\end{array}\right), \quad P_{1}:=\left(\begin{array}{ll}
0 & 1 \\
1 & 0
\end{array}\right)
$$

and $P_{0}:=0 \in \mathbb{R}^{2 \times 2}$, the pde (5.1) takes the form (2.1) of a port-Hamiltonian system of order $N=1$ and, moreover, the boundary condition (5.2) and the in- and output conditions (5.3) take the desired form (2.6) and (2.2), (2.7) with matrices $W_{B, 1}, W_{B, 2}, W_{C} \in \mathbb{R}^{1 \times 4}$. It is straightforward to verify that this system $\mathfrak{S}$ is impedance-energy-preserving, that the matrix $W \in \mathbb{R}^{3 \times 4}$ from (3.2) has full rank, and that $\mathcal{H}$ is absolutely continuous and (4.6) holds true. In particular, Conditions 2.1, 3.1, 4.3 are satisfied. So, as soon as the controller $\mathfrak{S}_{c}$ is chosen such that

- Conditions 2.2 and 4.4 are satisfied, or

- Conditions 2.2 and 4.10 are satisfied and 0 is the only critical point of $\mathcal{P}$,

respectively, the resulting closed-loop system $\tilde{\mathfrak{S}}$ is input-to-state stable or weakly input-to-state stable, respectively (Lem. 4.16 and 4.17).

Example 5.2. Consider a beam modelled according to Timoshenko $[2,12,53]$, that is, the transverse displacement $w(t, \zeta)$ and the rotation angle $\varphi(t, \zeta)$ of the beam at position $\zeta \in(a, b)$ evolve according to the partial differential equations

$$
\begin{gathered}
\rho(\zeta) \partial_{t}^{2} w(t, \zeta)=\partial_{\zeta}\left(K(\zeta)\left(\partial_{\zeta} w(t, \zeta)-\varphi(t, \zeta)\right)\right) \\
I_{r}(\zeta) \partial_{t}^{2} \varphi(t, \zeta)=\partial_{\zeta}\left(E I(\zeta) \partial_{\zeta} \varphi(t, \zeta)\right)+K(\zeta)\left(\partial_{\zeta} w(t, \zeta)-\varphi(t, \zeta)\right)
\end{gathered}
$$

for $t \in[0, \infty), \zeta \in(a, b)$ (Timoshenko beam equations) and the energy $E_{w, \varphi}(t)$ of the beam at time $t$ is given by

$$
E_{w, \varphi}(t)=\frac{1}{2} \int_{a}^{b} \rho(\zeta)\left(\partial_{t} w(t, \zeta)\right)^{2}+K(\zeta)\left(\partial_{\zeta} w(t, \zeta)-\varphi(t, \zeta)\right)^{2}+I_{r}(\zeta)\left(\partial_{t} \varphi(t, \zeta)\right)^{2}+E I(\zeta)\left(\partial_{\zeta} \varphi(t, \zeta)\right)^{2} \mathrm{~d} \zeta
$$

In these equations, $\rho, E, I, I_{r}, K$ are respectively the mass density, the Young modulus, the moment of inertia, the rotatory moment of inertia, and the shear modulus of the beam and they are assumed to be absolutely continuous and to be bounded below and above by positive finite constants. Also, assume that the beam is clamped at its left end, that is,

$$
\partial_{t} w(t, a)=0 \quad \text { and } \quad \partial_{t} \varphi(t, a)=0 \quad(t \in[0, \infty))
$$

(velocity and angular velocity at the left endpoint $a$ are zero), and that the control input $u(t)$ is given by the force and the torsional moment at the right end of the beam and the observation output $y(t)$ is given by the velocity and angular velocity at the right end of the beam, that is,

$$
u(t)=\left(\begin{array}{c}
K(b)\left(\partial_{\zeta} w(t, b)-\varphi(t, b)\right) \\
E I(b) \partial_{\zeta} \varphi(t, b)
\end{array}\right), \quad y(t)=\left(\begin{array}{c}
\partial_{t} w(t, b) \\
\partial_{t} \varphi(t, b)
\end{array}\right)
$$


for all $t \in[0, \infty)$. With the choices

$$
x(t)(\zeta):=\left(\begin{array}{c}
\partial_{\zeta} w(t, \zeta)-\varphi(t, \zeta) \\
\rho(\zeta) \partial_{t} w(t, \zeta) \\
\partial_{\zeta} \varphi(t, \zeta) \\
I_{r}(\zeta) \partial_{t} \varphi(t, \zeta)
\end{array}\right), \quad \mathcal{H}(\zeta):=\left(\begin{array}{cccc}
K(\zeta) & 0 & 0 & 0 \\
0 & 1 / \rho(\zeta) & 0 & 0 \\
0 & 0 & E I(\zeta) & 0 \\
0 & 0 & 0 & 1 / I_{r}(\zeta)
\end{array}\right)
$$

and an appropriate choice of $P_{1}, P_{0} \in \mathbb{R}^{4 \times 4}$, the pde (5.4) take the form (2.1) of a port-Hamiltonian system of order $N=1$ and, moreover, the boundary condition (5.6) and the in- and output conditions (5.7) take the desired form (2.6) and (2.2), (2.7) with matrices $W_{B, 1}, W_{B, 2}, W_{C} \in \mathbb{R}^{2 \times 8}$. It is straightforward to verify that this system $\mathfrak{S}$ is impedance-energy-preserving, that the matrix $W \in \mathbb{R}^{6 \times 8}$ from (3.2) has full rank, and that $\mathcal{H}$ is absolutely continuous and (4.6) holds true. In particular, Conditions 2.1, 3.1, 4.3 are satisfied. So, as soon as the controller $\mathfrak{S}_{c}$ is chosen such that

- Conditions 2.2 and 4.4 are satisfied, or

- Conditions 2.2 and 4.10 are satisfied and 0 is the only critical point of $\mathcal{P}$,

respectively, the resulting closed-loop system $\tilde{\mathfrak{S}}$ is input-to-state stable or weakly input-to-state stable, respectively (Lem. 4.16 and 4.17).

Acknowledgements. J. Schmid gratefully acknowledges financial support from the German Research Foundation (DFG) through the research training group "Spectral theory and dynamics of quantum systems" (GRK 1838) and through the grant "Input-to-state stability and stabilization of distributed-parameter systems" (DA 767/7-1).

\section{REFERENCES}

[1] B. Augner, Well-posedness and stability of linear port-Hamiltonian systems with nonlinear boundary feedback. SIAM $J$. Control Optim. 57 (2019) 1818-1844.

[2] B. Augner, Stabilisation of infinite-dimensional port-Hamiltonian systems via dissipative boundary feedback. PhD. thesis, Universität Wuppertal (2016).

[3] G. Bastin and J.-M. Coron, Stability and boundary stabilization of 1-D hyperbolic systems. Birkhäuser (2016).

[4] F.H. Clarke, Y.S. Ledyaev and R.J. Stern, Asymptotic stability and smooth Lyapunov functions. J. Differ. Equ. 149 (1998) 69-114.

[5] R. Curtain and H. Zwart, Introduction to infinite-dimensional systems theory. A state-space approach. Springer (2020)

[6] S. Dashkovskiy and A. Mironchenko, Input-to-state stability of infinite-dimensional control systems. Math. Contr. Sign. Syst. 25 (2013) 1-35

[7] S. Dashkovskiy, A. Mironchenko, J. Schmid and F. Wirth, Stability of infinitely many interconnected systems. Conference proceedings of the 11th IFAC Symposium on Nonlinear Control Systems, IFAC-PapersOnLine 52 (2019) 937-942

[8] S. Dashkovskiy, O. Kapustyan and J. Schmid, A local input-to-state stability result w.r.t. attractors of nonlinear reactiondiffusion equations. Math. Contr. Sign. Syst. 32 (2020) 309-326.

[9] V. Duindam, A. Macchelli, S. Stramigioli and H. Bruyninckx (editors), Modeling and control of complex physical systems the port-Hamiltonian approach. Springer (2009).

[10] M.S. Edalatzadeh and K. Morris, Stability and well-posedness of a nonlinear railway track model. IEEE Control Syst. Lett. 3 (2019).

[11] H.O. Fattorini, Boundary control systems. SIAM J. Contr. 6 (1968) 349-388.

[12] B. Jacob and H. Zwart, Linear port-Hamiltonian systems on infinite-dimensional spaces. Birkäuser (2012).

[13] B. Jacob, K. Morris and H. Zwart, $C_{0}$-semigroups for hyperbolic partial differential equations on a one-dimensional spatial domain. J. Evol. Equ. 15 (2015) 493-502.

[14] B. Jacob and J. Kaiser, Well-posedness of networks for 1-D hyperbolic partial differential equations. J. Evol. Equ. 19 (2019) 91-109.

[15] B. Jacob, R. Nabiullin, J. Partington and F. Schwenninger, Infinite-dimensional input-to-state stability and Orlicz spaces. SIAM J. Contr. Optim. 56 (2018) 868-889.

[16] B. Jacob and F. Schwenninger, Input-to-state stability of unbounded bilinear control systems. arXiv:1811.08470 (2018).

[17] B. Jacob, F.L. Schwenninger and H. Zwart, On continuity of solutions for parabolic control systems and input-to-state stability. J. Differ. Equ. 266 (2018) 6284-6306.

[18] B. Jacob, A. Mironchenko, J.R. Partington and F. Wirth, Non-coercive Lyapunov functions for input-to-state stability of infinite-dimensional systems. SIAM J. Contr. Optim. 58 (2020) 2952-2978. 
[19] B. Jacob, F. Schwenninger and L. Vorberg, Remarks on input-to-state stability of collocated systems with saturated feedback. arXiv:2001.01636 (2020).

[20] B. Jayawardhana and G. Weiss, State convergence of poassive nonlinear systems with $L^{2}$ input. IEEE Trans. Autom. Contr. 54 (2009) 1723-1727.

[21] H.G. Kankanamalage, Y. Lin and Y. Wang, On Lyapunov-Krasovskii characterizations of input-to-output stability. IFACPapersOnLine 50 (2017) 14362-14367.

[22] I. Karafyllis and M. Krstic, ISS with respect to boundary disturbances for 1-D parabolic PDEs. IEEE Trans. Automat. Contr. 61 (2016) 3712-3724.

[23] I. Karafyllis and M. Krstic, ISS in different norms for 1-D parabolic PDEs with boundary disturbances. SIAM J. Contr. Optim. 55 (2017) 1716-1751.

[24] I. Karafyllis and M. Krstic, Input-to-State Stability for PDEs. Springer (2019).

[25] C. Kawan, A. Mironchenko, A. Swikir, N. Noroozi and M. Zamani, A Lyapunov-based ISS small-gain theorem for infinite networks. IEEE Trans. Autom. Contr. (2019) doi: 10.1109/TAC.2020.3042410.

[26] Y. Le Gorrec, H. Zwart and B. Maschke, Dirac structures and boundary control systems associated with skew-symmetric differential operators. SIAM J. Contr. Optim. 44 (2005) 1864-1892.

[27] F. Mazenc and C. Prieur, Strict Lyapunov functions for semilinear parabolic partial differential equations. Math. Contr. Rel. Fields 1 (2011) 231-250.

[28] M. Miletic, D. Stürzer, A. Arnold and A. Kugi, Stability of an Euler-Bernoulli beam with a nonlinear dynamic feedback system. IEEE Trans. Autom. Contr. 61 (2016) 2782-2795.

[29] A. Mironchenko, Local input-to-state stability: characterizations and counterexamples. Syst. Contr. Lett. 87 (2016) $23-28$.

[30] A. Mironchenko and F. Wirth, Characterizations of input-to-state stability for infinite-dimensional systems. IEEE Trans. Autom. Contr. 63 (2018) 1692-1707.

[31] A. Mironchenko and F. Wirth, Lyapunov characterization of input-to-state stability for semilinear control systems over Banach spaces. Syst. Contr. Lett. 119 (2018) 64-70.

[32] A. Mironchenko, I. Karafyllis and M. Krstic, Monotonicity methods for input-to-state stability of nonlinear parabolic PDEs with boundary disturbances. SIAM J. Contr. Optim. 57 (2019) 510-532.

[33] A. Mironchenko, Criteria for input-to-state practical stability. IEEE Trans. Autom. Contr. 64 (2019) $298-304$.

[34] A. Mironchenko, Small gain theorems for general networks of heterogeneous infinite-dimensional systems. arXiv:1901.03747 (2019).

[35] A. Mironchenko, C. Kawan and J. Glück, Nonlinear small-gain theorems for input-to-state stability of infinite interconnections. arXiv:2007.05705 (2020).

[36] A. Mironchenko and C. Prieur, Input-to-state stability of infinite-dimensional systems: recent results and open questions. SIAM Rev. 62 (2020) 529-614.

[37] R. Nabiullin and F. Schwenninger, Strong input-to-state stability for infinite dimensional linear systems. Math. Contr. Sign. Syst. 30 (2018).

[38] J. Oostveen, Strongly stabilizable distributed parameter systems. SIAM Frontiers Appl. Math. (2000).

[39] A. Pazy, Semigroups of linear operators and applications to partial differential equations. Springer (1983).

[40] P. Pepe, On Liapunov-Krasovskii functionals under Caratheodory conditions. Automatica 43 (2007) 701-706.

[41] H. Ramirez, Y. Le Gorrec, A. Macchelli and H. Zwart, Exponential stabilization of boundary controlled port-Hamiltonian systems with dynamic feedback. IEEE Trans. Autom. Contr. 59 (2014) 2849-2855.

[42] H. Ramirez, H. Zwart and Y. Le Gorrec, Stabilization of infinite-dimensional port-Hamiltonian systems by nonlinear dynamic boundary control. Automatica $\mathbf{8 5}$ (2017) 61-69.

[43] J. Schmid and H. Zwart, Stabilization of port-Hamiltonian systems by nonlinear boundary control in the presence of disturbances. Conference proceedings of the 23rd Symposium on Mathematical Theory of Networks and Systems (2018) $570-575$.

[44] J. Schmid, Stabilization of port-Hamiltonian systems with discontinuous energy densities. arXiv:1809.00857 (2018). Accepted provisionally in Evol. Equ. Contr. Th.

[45] J. Schmid, Weak input-to-state stability: characterizations and counterexamples. Math. Contr. Sign. Syst. 31 (2019) 433-454.

[46] J. Schmid, S. Dashkovskiy, B. Jacob and H. Laasri, Well-posedness of non-autonomous semilinear systems. Conference proceedings of the 11th Symposium on Nonlinear Control Systems, IFAC-PapersOnLine 52 (2019) 216-220.

[47] J. Schmid, Well-posedness and stability of non-autonomous semilinear input-output systems. arXiv:1904.10376 (2019). Accepted provisionally in Evol. Equ. Contr. Th.

[48] J. Schmid, Infinite-time admissibility under compact perturbations. In: J. Kerner, H. Laasri, D. Mugnolo (eds.), Control theory of infinite-dimensional systems, Operator Theory: Advances and Applications 277 (2020) 73-82.

[49] J. Schmid, O. Kapustyan and S. Dashkovskiy, Asymptotic gain results for attractors of semilinear systems. arXiv:1909.06302 (2019). Accepted for publication in Math. Contr. Rel. Fields

[50] F. Schwenninger, Input-to-state stability for parabolic boundary control: linear and semi-linear systems. In: J. Kerner, H. Laasri, D. Mugnolo (eds.), Control theory of infinite-dimensional systems, Operator Theory: Advances and Applications 277 (2020) 83-116.

[51] A. Tanwani, C. Prieur and S. Tarbouriech, Disturbance-to-state stabilization and quantized control for linear hyperbolic systems. arXiv:1703.00302 (2017).

[52] M. Tucsnak and G. Weiss, Well-posed systems - the LTI case and beyond. Automatica 50 (2014) $1757-1779$. 
[53] J. Villegas, A port-Hamiltonian approach to distributed-parameter systems. Ph.D. thesis, Universiteit Twente (2007).

[54] J. Villegas, H. Zwart, Y. Le Gorrec and B. Maschke, Exponential stability of a class of boundary control systems. IEEE Trans. Autom. Contr. 54 (2009) 142-147.

[55] G. Weiss, Admissibility of unbounded control operators. SIAM J. Contr. Optim. 27 (1989) 527-545.

[56] J. Zheng and G. Zhu, Input-to-state stability with respect to boundary disturbances for a class of semi-linear parabolic equations. Automatica 97 (2018) 271-277.

[57] J. Zheng and G. Zhu, A De Giorgi iteration-based approach for the establishment of ISS properties for burgers' equation with boundary and in-domain disturbances. IEEE Trans. Automat. Contr. 64 (2019) 3476-3483.

[58] J. Zheng and G. Zhu, A weak maximum principle-based approach for input-to-state stability of nonlinear parabolic PDEs with boundary disturbances. Math. Control Signals Syst. 32 (2020) 157-176.

[59] H. Zwart, H. Ramirez and Y. Le Gorrec, Asymptotic stability for a class of boundary control systems with non-linear damping. In: The second IFAC workshop on control of systems governed by partial differential equations (CPDE). Bertinoro, Italy (2016). 